\title{
An efficient one-pot three-component synthesis of tetrakis(uracil) and their corresponding bis-fused derivatives
}

\author{
Amr M. Abdelmoniem, ${ }^{a}$ Said A. S. Ghozlan, ${ }^{a}$ Holger Butenschön, ${ }^{b}$ Doaa M. Abdelmoniem, ${ }^{a}$ \\ Ahmed H. M. Elwahy, ${ }^{* a}$ and Ismail A. Abdelhamid*a \\ a Department of Chemistry, Faculty of Science, Cairo University,12613 Giza, A. R. Egypt
}

${ }^{b}$ Institut für Organische Chemie, Leibniz Universität Hannover, Schneiderberg 1B, 30167 Hannover, Germany

Email: aelwahy@hotmail.com, ismail shafy@yahoo.com

Received 01-07-2019

Accepted 03-03-2019

Published on line 03-17-2019

\section{Abstract}

A concise and efficient approach to tetrakis(uracil) derivatives by the reaction of bis(aldehydes) with four equivalents of 6 -aminouracil is reported. Also, the synthesis of bis(pyrimido[4,5-b]quinolones) and bis(indeno$\left[2^{\prime}, 1^{\prime}: 5,6\right]$ pyrido[2,3-d]pyrimidine) derivatives has been accomplished by a three-component reaction involving bis(aldehydes), 6-aminouracil and the appropriate cyclic 1,3-diketone. The method involves domino Knoevenagel condensation / Michael addition reaction sequences.

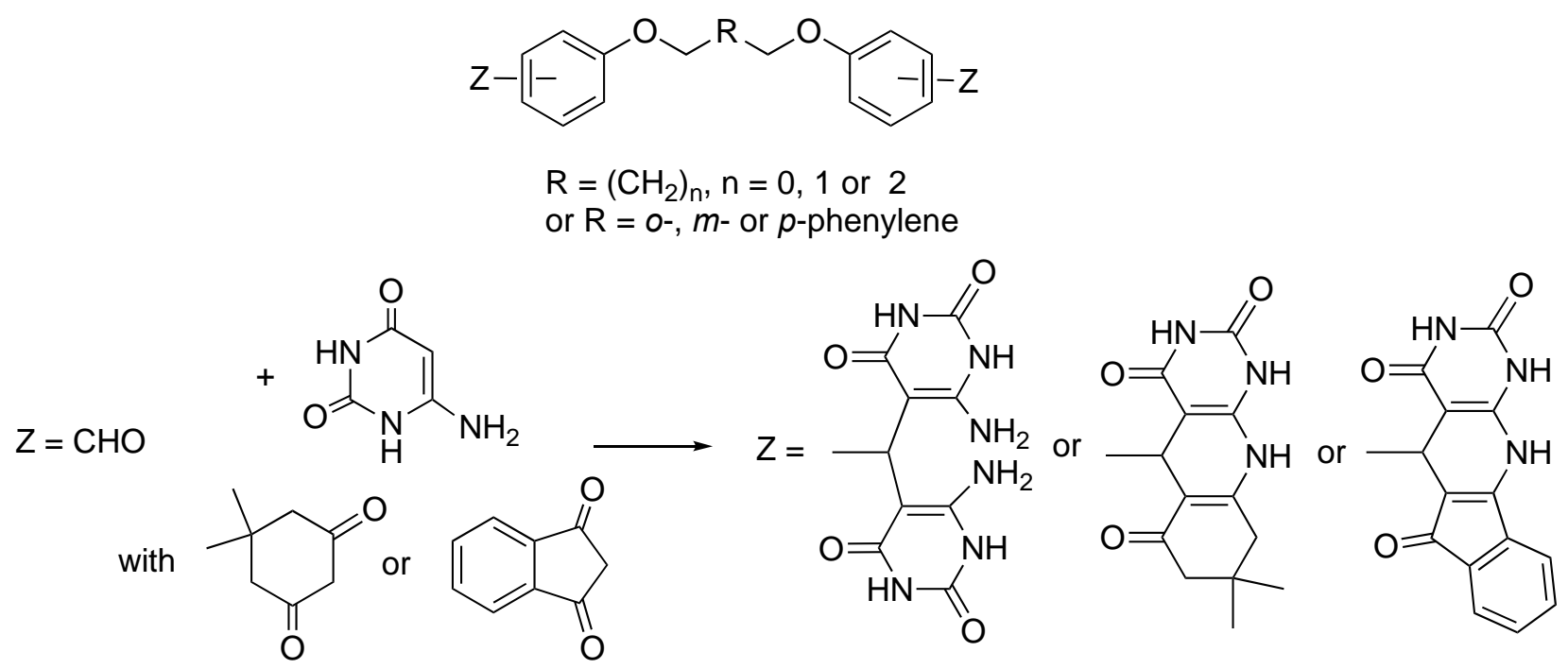

Keywords: Bis(aldehydes), Michael addition, uracils, fused pyrimidines, quinolinones, indenones 


\section{Introduction}

Uracil is a common and naturally occurring pyrimidine derivative and is one of the four nucleobases in the biopolymer RNA. ${ }^{1-5}$ The uracil scaffold and its derivatives exhibit a wide range of biological activities, including treatment of cancer and viral diseases. ${ }^{6-8}$ The annelated derivatives have also several applications as bronchodilators, ${ }^{9,10}$ antiviral agents, ${ }^{11}$ antiallergic compounds, ${ }^{9,10}$ adenosine receptor antagonists ${ }^{12,13}$ and antihypertensive agents. ${ }^{14}$ Uracil derivatives have also been used as versatile building blocks for the synthesis of a variety of heterocycles, including pyrimido[4,5- $d$ ]pyrimidines, ${ }^{15}$ pyrido[2,3- $d$ ]pyrimidines, ${ }^{15,16}$ pyrimido$[4,5-b]$ quinolines, ${ }^{16-21}$ and indeno[2',1':5,6]pyrido[2,3- $d$ ]pyrimidines. ${ }^{16,19,20}$ Pyrimido[4,5- $\left.b\right]$ quinolines have received much attention over the past years due to their wide range of applications including their use as anticancer 22,23 and as radioprotective ${ }^{22}$ agents. Furthermore, multicomponent reactions (MCRs) represent an important attractive synthetic strategy as they provide a rapid access to organic compounds of high structural complexity. MCRs offer the advantage of selectivity, simplicity, atom-economy and an overall efficiency much more than conventional chemical reactions. ${ }^{24-28}$ Keeping in mind the importance of the uracil moiety and in continuation of our work on multicomponent reactions, ${ }^{29-31}$ Michael addition, ${ }^{32-35}$ Hantzsch reactions ${ }^{31,36,37}$ as well as on the synthesis of bis-heterocycles, ${ }^{29-31,36,37}$ we report herein a highly efficient one-pot synthesis of tetrakis(uracil), bis(pyrimido[4,5-b]quinolones) and bis(indeno[2',1':5,6]pyrido[2,3- $d$ ]pyrimidine) derivatives.

\section{Results and Discussion}

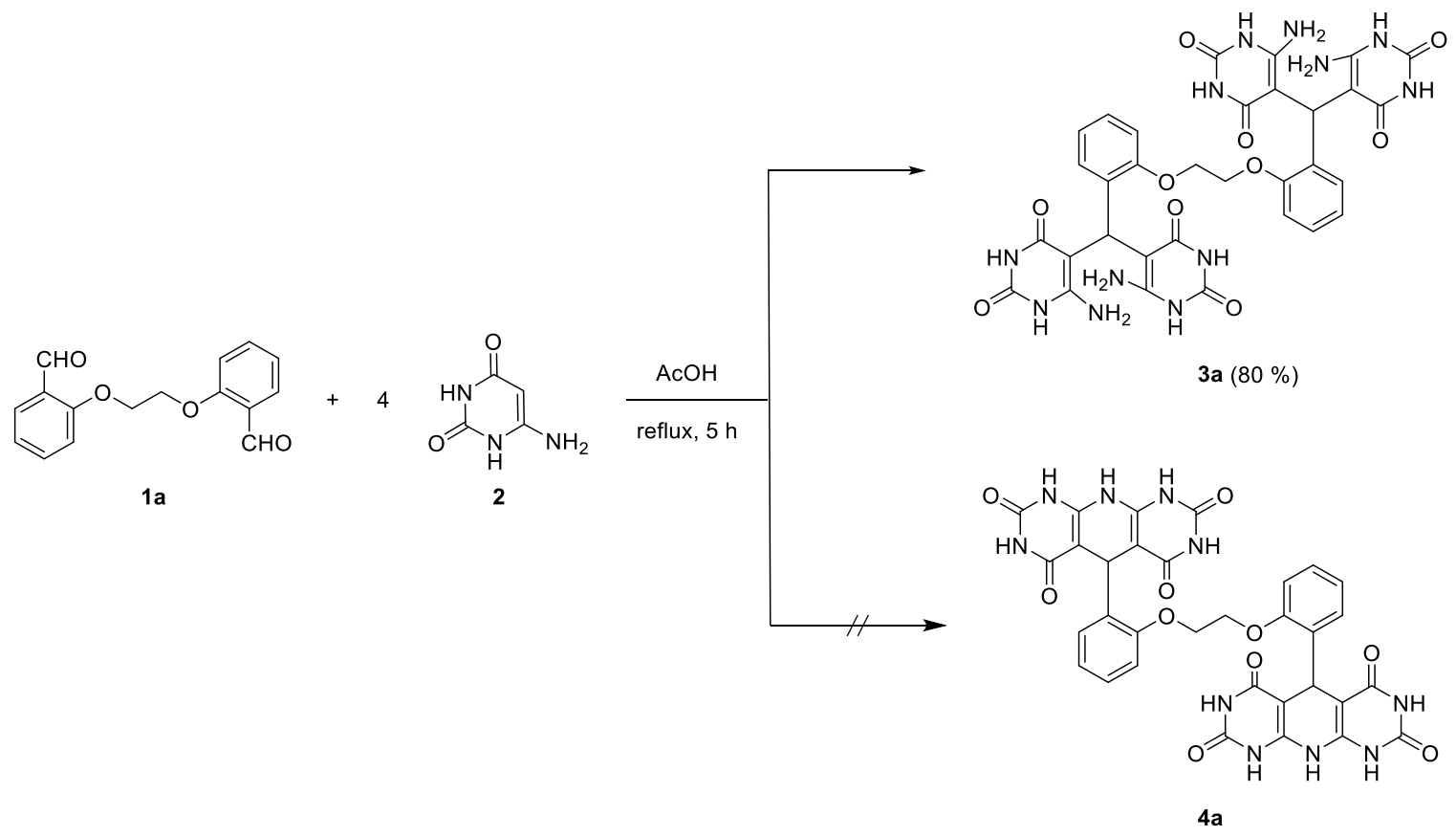

Scheme 1. The reaction of bis(aldehyde) 1a with 6-aminouracil 2 in acetic acid at reflux.

Firstly, we investigated the reaction of bis(aldehyde) 1a with 6-aminouracil $\mathbf{2}$ in acetic acid at reflux aiming at the synthesis of bis(9,10-dihydropyrido[2,3-d:6,5-d']dipyrimidinetetraone) 4a. Contrary to our expectation, the reaction did not yield compound $4 \mathrm{a}$ and instead gave the tetrakis(6-aminopyrimidine-2,4(1H,3H)-dione) $3 a$ in $80 \%$ yield (Scheme 1 ). 
Using a similar approach, reaction of aminouracil $\mathbf{2}$ with the appropriate bis(aldehyde)s $\mathbf{1 b}$-e afforded the corresponding tetrakis(uracils) 3b-e, in which the tetra-uracil units are linked to alkyl spacer via phenoxy groups, in $75-84 \%$ yields (Scheme 2 ).

Evidence for these structures came, for example for compound 3e, from the IR spectrum that indicated the presence of $\mathrm{NH}_{2}$ and $\mathrm{NH}$ groups at $v 3407,3181 \mathrm{~cm}^{-1}$. In addition, it revealed two different carbonyl groups at $\tilde{v} 1711$ and $1624 \mathrm{~cm}^{-1}$. The ${ }^{1} \mathrm{H}$ NMR spectrum of $3 \mathrm{e}$ indicated the presence of a multiplet signal integrating for four protons at $\delta 3.97 \mathrm{ppm}$ assigned to the two $\mathrm{OCH}_{2}$ groups, in addition to a singlet signal at $\delta 5.26$ characteristic for the two methine protons. Moreover, signals assigned to the two amino groups appear as broad signals at $\delta 6.64 \mathrm{ppm}$. The signals of the $\mathrm{NH}$ groups appear as broad singlets at $\delta 10.29$ and $10.48 \mathrm{ppm}$. The ${ }^{13} \mathrm{C}$ NMR spectrum of $3 \mathrm{e}$ is in agreement with the proposed structure. The particularly distinct signals at $\delta$ $26.1, \delta 32.0, \delta 67.5$ and $\delta 68.2$ are readily related to $\mathrm{OCH}_{2} \mathrm{CH}$, methine $\mathrm{CH}, \mathrm{OCH}_{2}$ and $\mathrm{C}-5$ of pyrimidinone moiety, respectively. The mass spectrum of compound 3 e exhibits the correct molecular ion peak at $\mathrm{m} / \mathrm{z} 770$ (3.5\%). The compound readily losses a CN moiety to give an ion at $m / z 744$ (2.6\%). The formation of peaks at $\mathrm{m} / \mathrm{z} 646(3.3 \%)$ and $508(2.9 \%)$ are characteristic for loss of uracil [M-uracil +1] and 2 uracil-CH [M-2 uracil-CH] fragments, respectively.
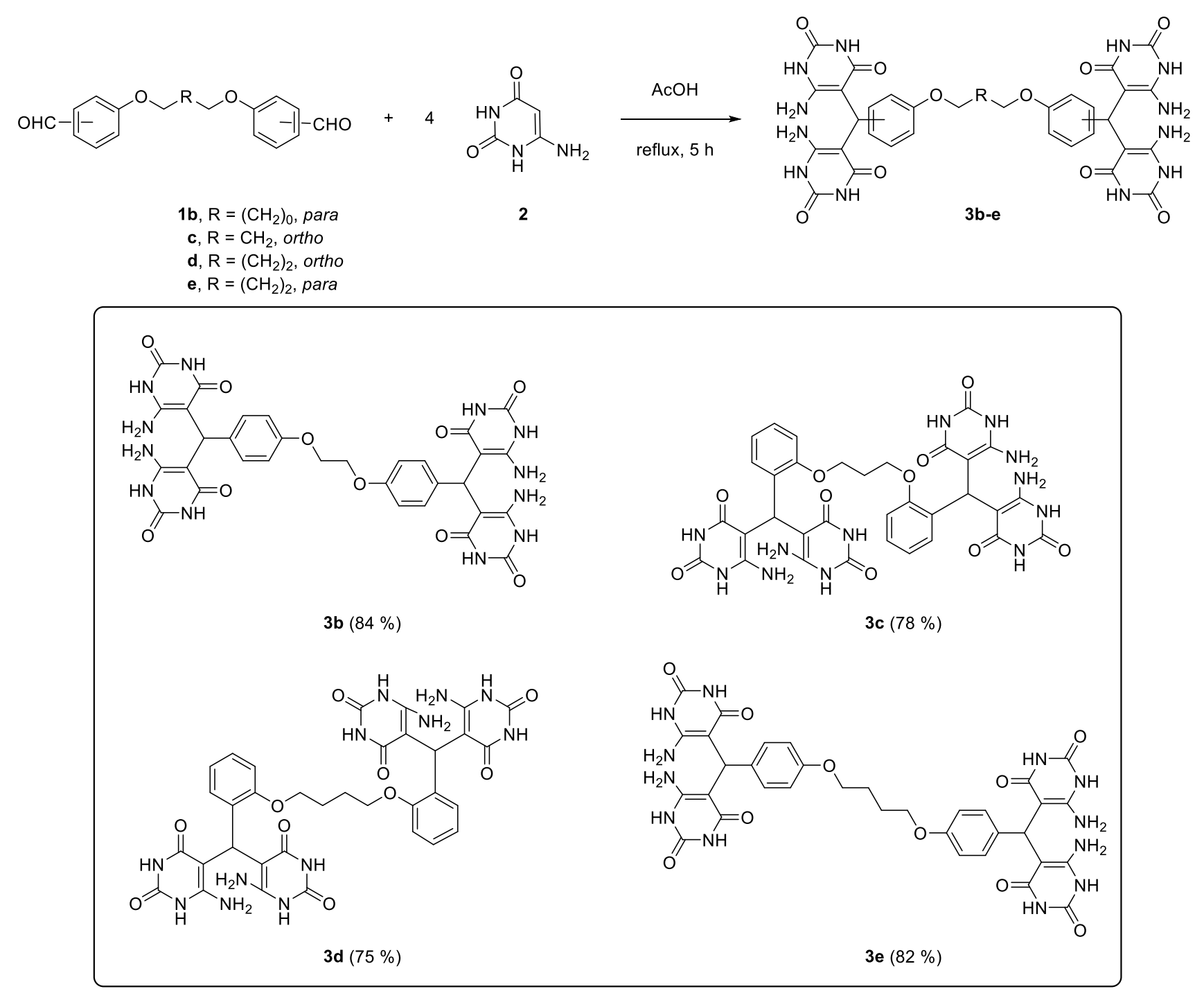

Scheme 2. Synthesis of tetrakis(uracils) 3b-e linked to alkyl spacer via a phenoxy group. 
Encouraged by the above results, and in a trial to ascertain the scope and generality of the protocol, a series of bis(aldehydes) containing aromatic linkages $\mathbf{1 f - 1 h}$, was used. Thus, the reaction of bis(aldehydes) $\mathbf{1 f}$ $\mathbf{1 h}$ with four equivalents of 6-aminouracil $\mathbf{2}$ afforded the tetrakis(uracil) derivatives $\mathbf{3 f}-\mathbf{3 h}$, which are linked to the benzene core via phenoxymethyl linkages in $73-76 \%$ yields (Scheme 3 ).

A proposed mechanism for the reaction is shown in Scheme 4. Nucleophilic addition of the enamine $\beta$ carbon of 6-aminouracil $\mathbf{2}$ to the two carbonyl centers of the bis(aldehydes) $\mathbf{1}$ affords the corresponding adducts 5. Subsequent elimination of water leads to the formation of the corresponding ene-imine intermediate $\mathbf{6}$. Michael addition of two molecules of $\mathbf{2}$ then react with $\mathbf{6}$ to afford the products $\mathbf{3}$ (Scheme 4 ).
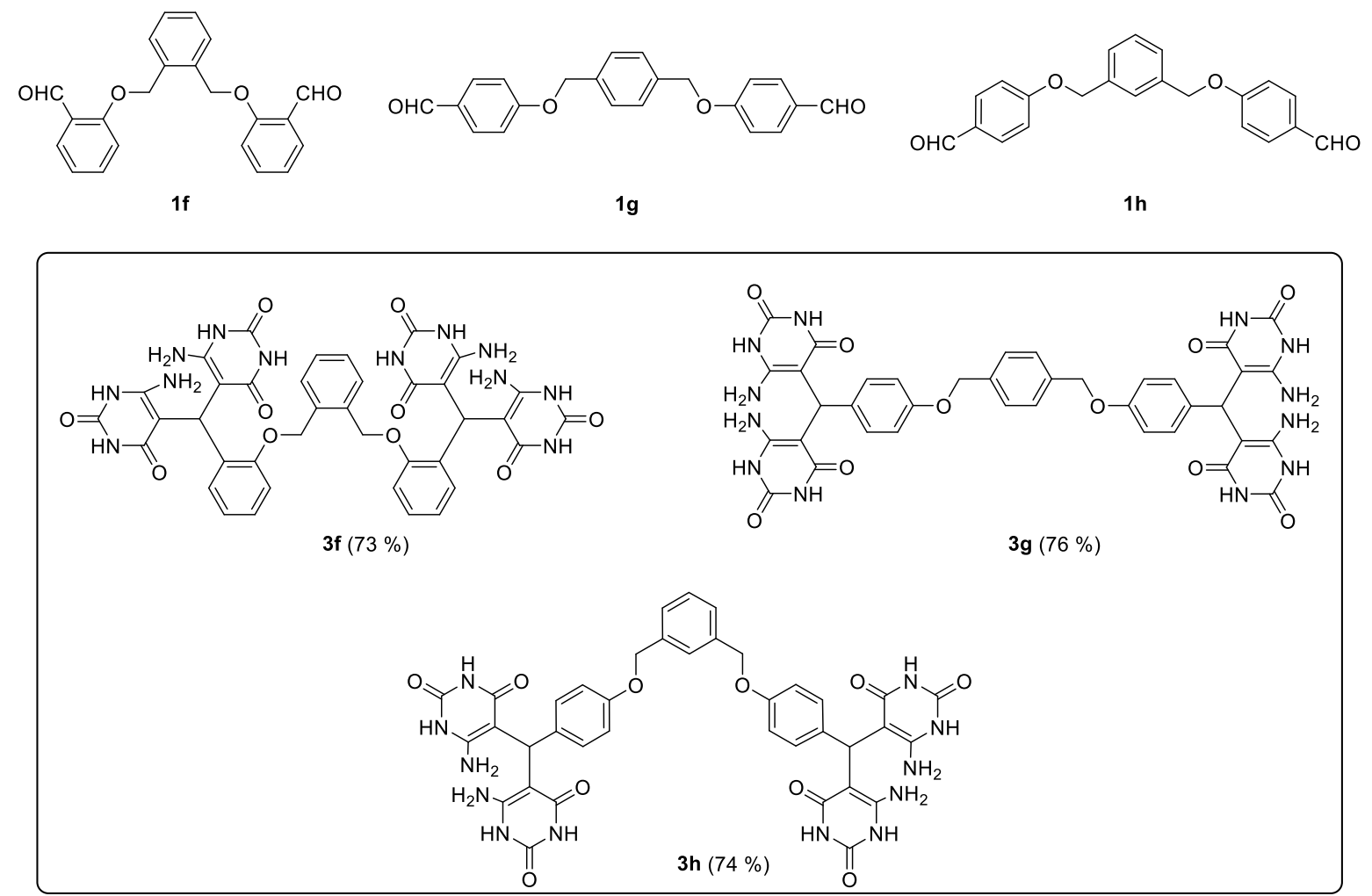

Scheme 3. Tetrakis(uracil) derivatives containing benzene core and phenoxymethyl linkages $\mathbf{3 f - 3 h}$. 

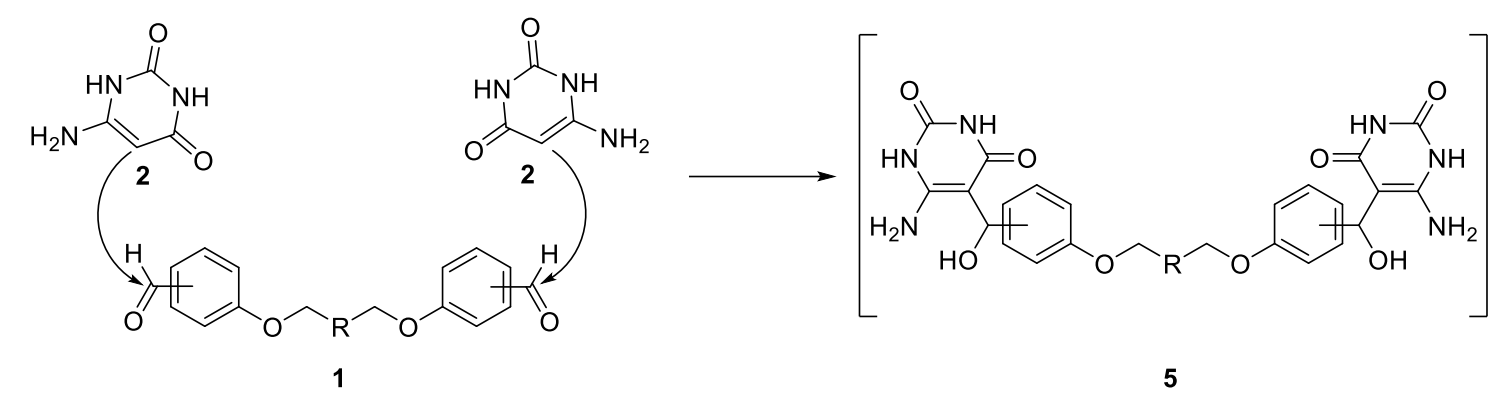

$\downarrow-\mathrm{H}_{2} \mathrm{O}$
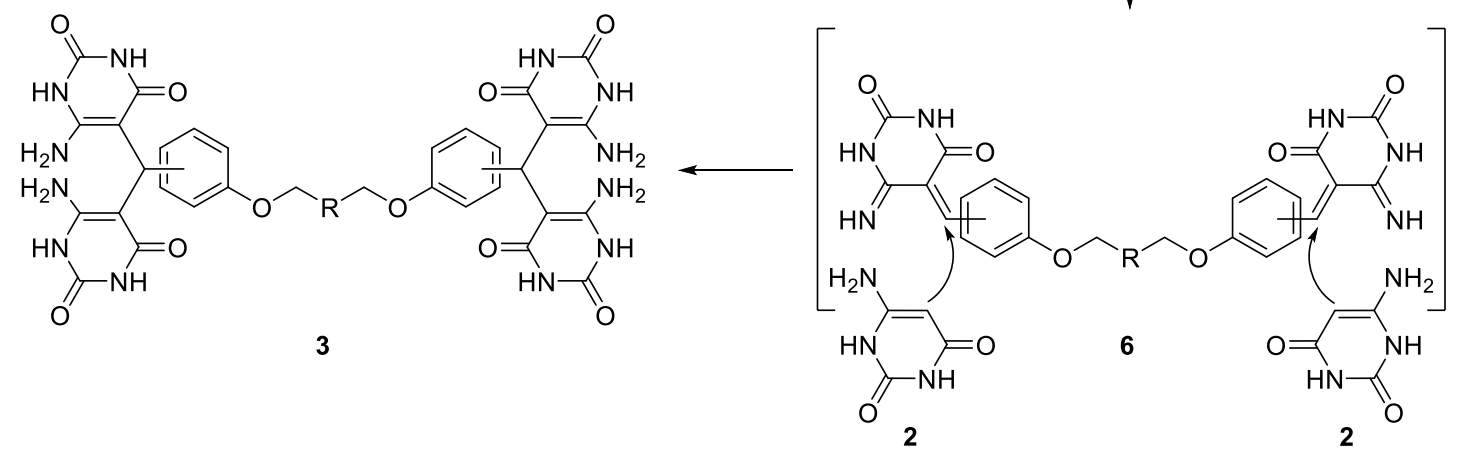

Scheme 4. A proposed mechanism for the synthesis of compounds 3.

Similarly, the three-component reaction of bis(aldehyde) 1a with two moles of either 6-aminouracil 2 or 5,5-dimethyl-1,3-cyclohexanedione 7 afforded the corresponding bis(pyrimido[4,5-b]quinolines) 9a in 83\% yield. In this case, uncyclized adduct 8 a was not obtained (Scheme 5).

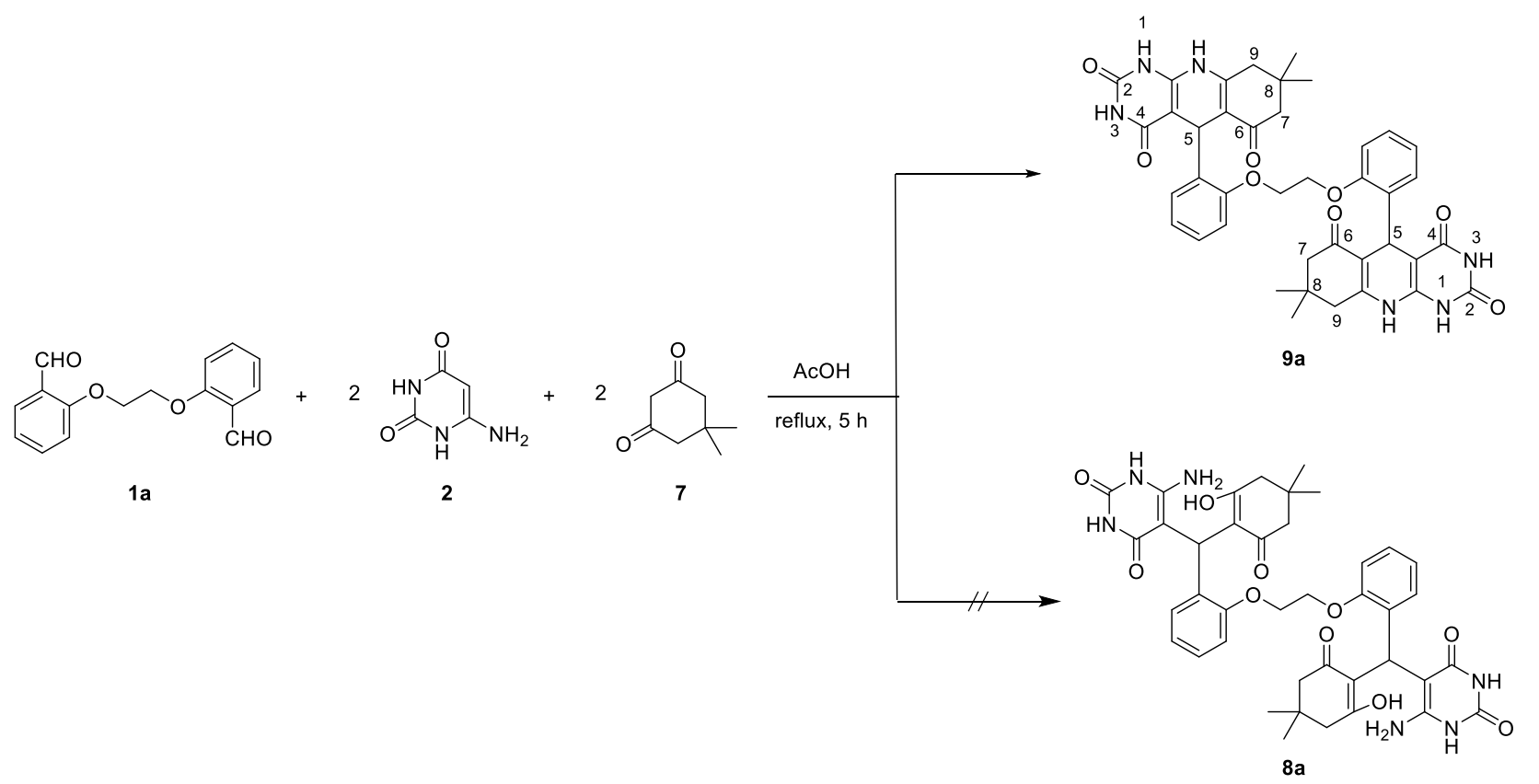

Scheme 5. A three-component reaction of bisaldehyde 1a, 6-aminouracil 2 and dimedone 7. 
The structure of compound 9a was established based on the elemental analyses and spectral data. The IR spectra indicated the presence of $\mathrm{NH}$ groups with absorption bands at $v 3424,3294$ and $3227 \mathrm{~cm}^{-1}$. In addition, it revealed a sharp band at $v 1721 \mathrm{~cm}^{-1}$ corresponding to the ketonic $\mathrm{C}=\mathrm{O}$ and a broad band at $v 1632 \mathrm{~cm}^{-1}$ for both amidic carbonyl groups $(\mathrm{C}=\mathrm{O}-2,4)$. The ${ }^{1} \mathrm{H}$ NMR spectrum of $9 \mathrm{a}$ indicated the presence of two singlets at $\delta 0.8$ and $0.99 \mathrm{ppm}$ assigned to the four methyl groups. In addition, a multiplet at $\delta 1.90-2.29$ ppm was assigned to $\mathrm{H} 7$ and $\mathrm{H} 9$. It also featured the diastereotopic methylene ether linkage $\mathrm{OCH}_{2}$ as a multiplet at $\delta$ 4.10-4.25 ppm. The singlet signal at $4.86 \mathrm{ppm}$ is assigned to $\mathrm{H} 5$. The spectrum also featured the aromatic protons as multiplets at $\delta 6.81-7.27 \mathrm{ppm}$. The $\mathrm{NH}$ groups appeared as three broad signals integrating for six protons at $\delta 8.98,10.11$ and 10.56 ppm. Similarly, bis(pyrimido[4,5-b]quinolines) 9b-d and $\mathbf{9 g}$-i, in which the pyrimido[4,5- $b$ ]quinoline moieties are linked to aliphatic or aromatic spacers, were successfully prepared in $82-89 \%$ yield by reaction of the appropriate bis-aldehyde $\mathbf{1} \mathbf{b}-\mathbf{d}$ and $\mathbf{1 g}$-i with two mole equivalents of both 6 aminouracil $\mathbf{2}$ and 5,5-dimethyl-1,3-cyclohexanedione $\mathbf{7}$ (Scheme 6). Compounds $\mathbf{9}$ should exist as a mixture of meso and $R, S$ diastereomers because there is no obvious reason for a diastereoselective reaction. Inspection of the ${ }^{1} \mathrm{H}$ and ${ }^{13} \mathrm{C}$ NMR spectra indicated that in most cases only one diastereomeric isomer (compounds 9a, $9 \mathrm{~b}, 9 \mathrm{~g}, 9 \mathrm{~h}$ and $9 \mathrm{i}$ ) was formed. However, in some cases, a mixture of meso and $R, S$ diastereomers (9c and $9 \mathrm{~d}$.) was apparently produced ( $c f$. experimental data).

A reasonable mechanistic pathway is shown in Scheme 7. The first step involves the Knoevenagel condensation of the bis(aldehydes) 1 and cyclic 1,3-diketone 5 to generate diadduct 10. Intermediate 10 acts as a Michael acceptor, while 6-aminouracil can be considered a Michael donor. The reaction of one mole of compound $\mathbf{1 0}$ with two moles of $\mathbf{2}$ affords the Michael diadduct $\mathbf{1 1}$. The intermediate $\mathbf{1 1}$ undergoes an intramolecular cyclization involving the nucleophilic addition of the amino to the carbonyl group, followed by dehydration to afford the final isolated products 9 via the intermediacy of 12 . 


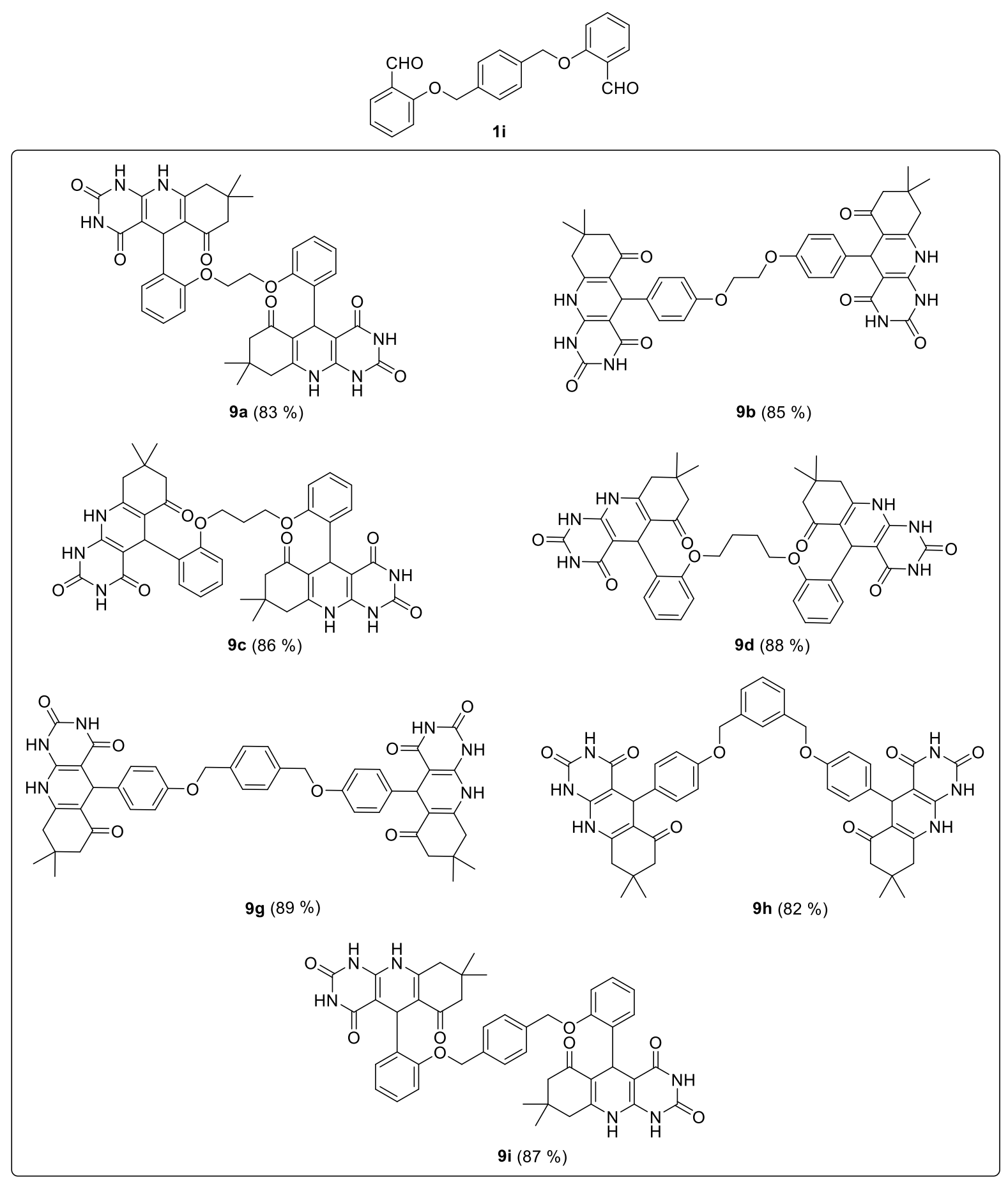

Scheme 6. Bis(pyrimido[4,5-b]quinolines) $9 \mathrm{~b}-\mathbf{d}$ and $\mathbf{9 g - i}$ with aliphatic or aromatic spacers. 


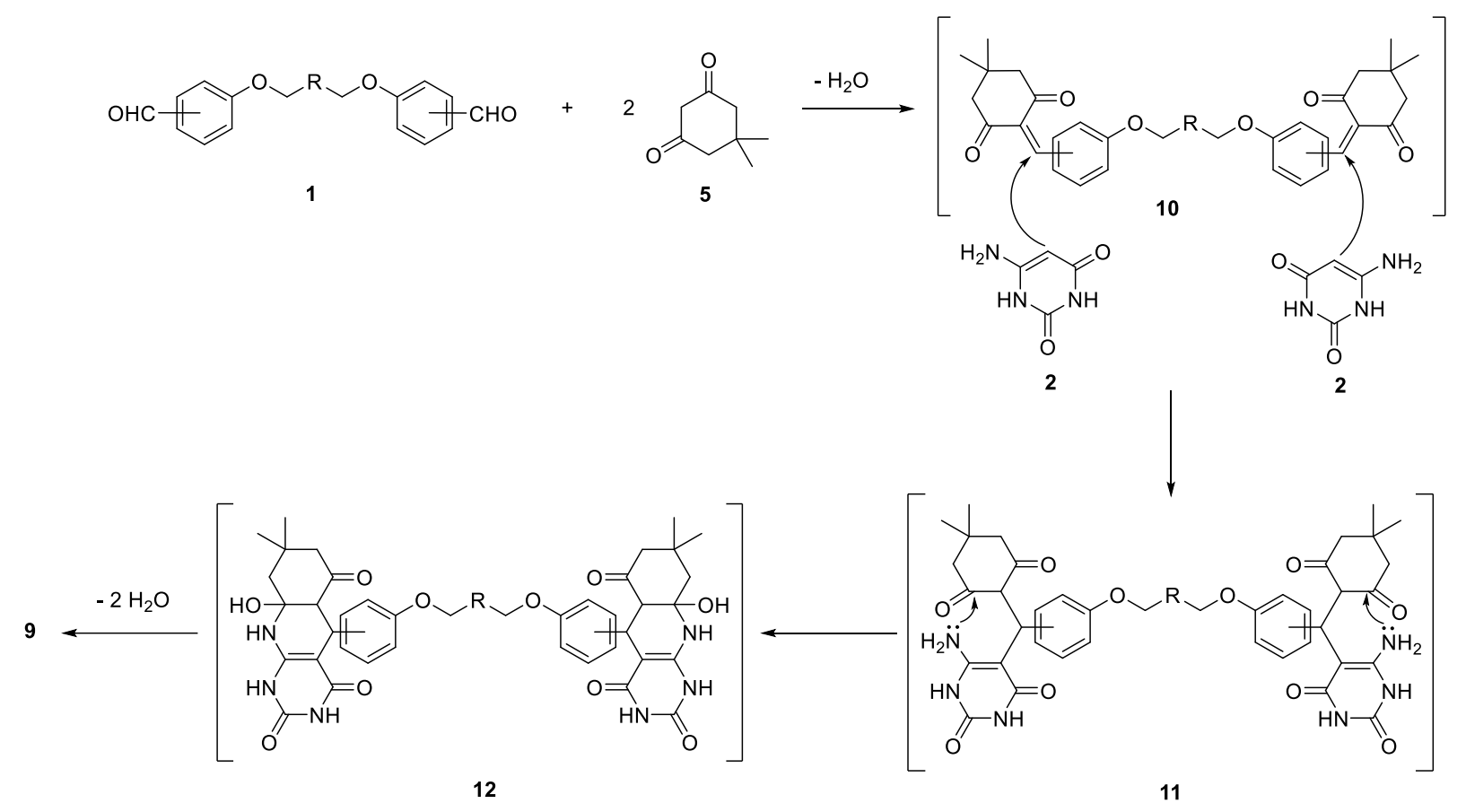

Scheme 7. A proposed mechanism for the synthesis of compounds $\mathbf{9 .}$
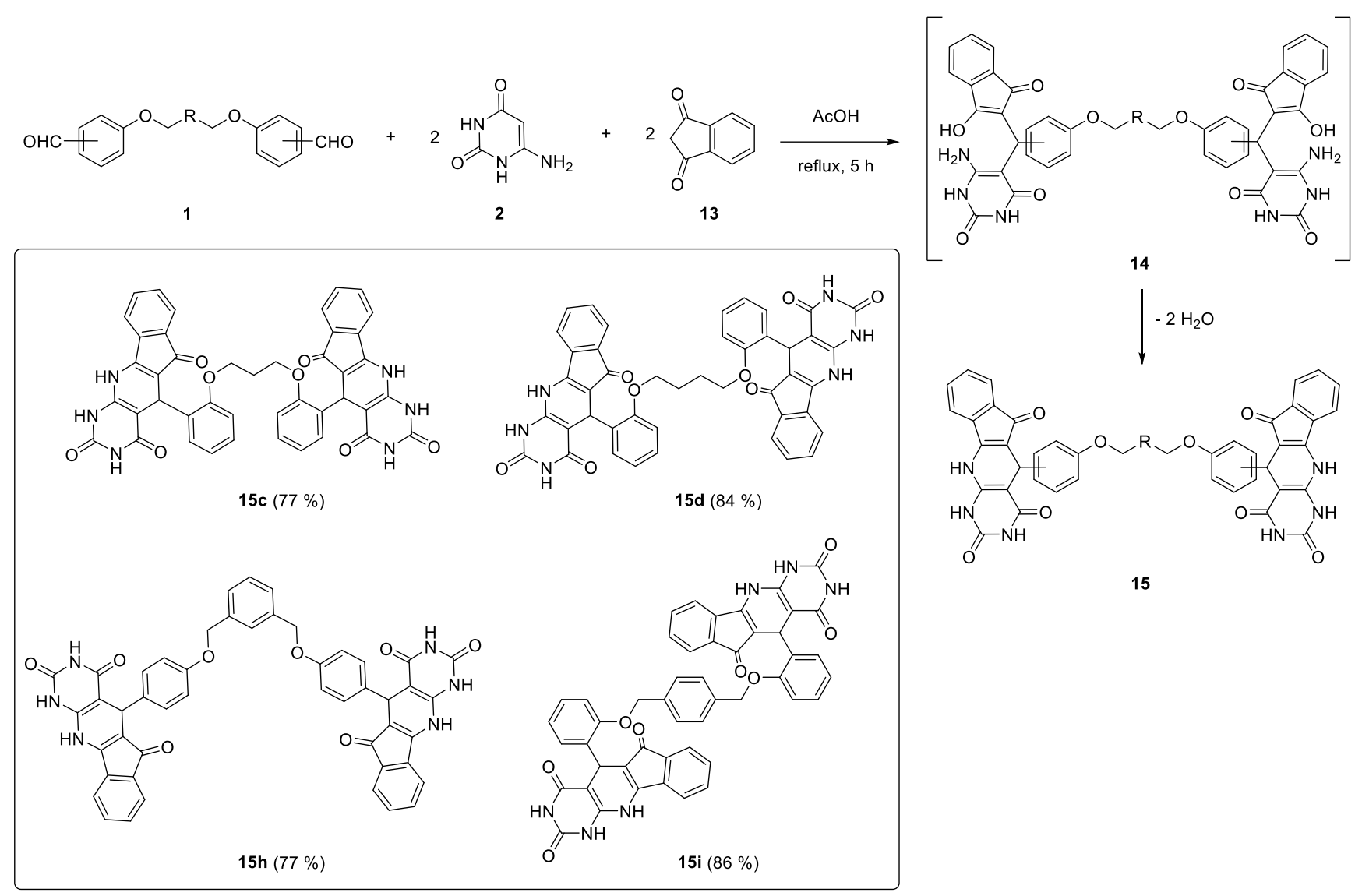

15

Scheme 8. Synthesis of bis(indeno[2',1':5,6]-pyrido[2,3-d]pyrimidines) 15.

In order to extend the scope of this reaction, the replacement of dimedone 7 with 1,3-indanedione 13 was investigated. Thus, under comparable reaction conditions, the condensation of bis(aldehydes) with two moles 
of both 6-aminouracil 2 and indanedione 13 proceeded smoothly and a new series of bis(indeno[2',1':5,6]pyrido[2,3-d]pyrimidines) 15 was obtained (Scheme 8). Similarly, as in the case of compounds $\mathbf{9}$, NMR spectra of compounds $\mathbf{1 5}$ indicated the formation of one compound in all cases except 15c, which gave a mixture of meso and $R, S$ diastereomers. One might assume that the stereogenic centers in 15c are more close to one another, so that the diastereomers may be distinguished, whereas in the other compounds, they are so far away from one another to show similar NMR signals (cf. experimental data).

\section{Conclusions}

In conclusion, we have demonstrated a simple and efficient route for the formation of tetrakis(uracil), bis(pyrimido[4,5-b]quinolones) and bis(indeno[2',1':5,6]pyrido[2,3- $d$ ]pyrimidine) derivatives by a threecomponent reaction involving bis(aldehydes), 6-aminouracil and the appropriate cyclic-1,3-dione.

\section{Experimental Section}

General. Melting points were measured with a Stuart melting point apparatus and are uncorrected. The IR spectra were recorded using a FTIR Bruker-vector 22 spectrophotometer as $\mathrm{KBr}$ pellets. The ${ }^{1} \mathrm{H}$ and ${ }^{13} \mathrm{C}$ NMR spectra were recorded in DMSO- $d_{6}$ as solvent at $400 \mathrm{MHz}$ and $100 \mathrm{MHz}$, respectively, on Bruker Ultrashield or Ascend NMR spectrometers. Chemical shifts are reported as $\delta$ values in ppm and referenced to the residual solvent signals as internal standards. Mass spectra were recorded with a Shimadzu GCMS-QP-1000 EX mass spectrometer in El $(70 \mathrm{eV})$ mode. The elemental analyses were performed at the Microanalytical center, Cairo University.

\section{Synthesis of compounds 3a-h. General procedure}

A mixture of bisaldehyde $1 \mathrm{a}-\mathrm{h}(1 \mathrm{mmol})$ and 6-aminouracil 2 (254 mg, $4 \mathrm{mmol}$ ) was heated at reflux in glacial $\mathrm{AcOH}(20 \mathrm{~mL})$ for $5 \mathrm{~h}$. The solvent was evaporated under reduced pressure. The residue was treated with aq. $\mathrm{NaHCO}_{3}$ solution $(0.5 \mathrm{~N}, 20 \mathrm{~mL})$, washed thoroughly with distilled $\mathrm{H}_{2} \mathrm{O}(20 \mathrm{~mL})$ and left to dry in air. The crude product was purified by crystallization from EtOH/1,4-dioxane (1:1, v/v, $10 \mathrm{~mL})$.

5,5',5",5"'-(((Ethane-1,2-diylbis(oxy))bis-(2,1-phenylene))bis(methanetriyl))tetrakis-(6-aminopyrimidine-

2,4(1H,3H)-dione) (3a). Yellowish white crystals (594 mg, 80\%), m. p. $>300{ }^{\circ} \mathrm{C} . \mathrm{IR}(\mathrm{KBr}): v_{\max } 3357,3181(\mathrm{br}$, $\mathrm{NH}_{2}$ and $\mathrm{NH}$ ), 1712 (CO), 1630 (CO) cm ${ }^{-1} .{ }^{1} \mathrm{H}$ NMR (400 MHz, DMSO-d 6 ): $\delta 4.26\left(\mathrm{~m}, 4 \mathrm{H}, 2 \mathrm{OCH}_{2} \mathrm{CH}_{2}\right), 5.32(\mathrm{~s}, 2$ $\mathrm{H}, 2 \mathrm{CH}-5), 6.61$ (br s, $8 \mathrm{H}, 4 \mathrm{NH}_{2}$ ), 6.77-6.91 (m, $\left.8 \mathrm{H}, \mathrm{Ar}-\mathrm{H}\right), 10.27$ (br s, $\left.2 \mathrm{H}, 2 \mathrm{NH}-1\right), 10.36$ (br s, $\left.2 \mathrm{H}, 4 \mathrm{NH}-3\right)$ ppm. ${ }^{13} \mathrm{C}$ NMR (100 MHz, DMSO-d $)$ : $\delta 31.9(\mathrm{CH}), 66.6\left(\mathrm{OCH}_{2}\right), 68.1(\mathrm{C5}), 114.0(\mathrm{Ar}-\mathrm{CH}), 121.4(\mathrm{Ar}-\mathrm{CH}), 124.5$ (Ar-C), $126.9(\mathrm{Ar}-\mathrm{CH}), 128.2(\mathrm{Ar}-\mathrm{CH}), 150.2(\mathrm{Ar}-\mathrm{C}), 155.6$ and 164.8 (CO-2 and CO-4) ppm. MS (El, $70 \mathrm{eV}): \mathrm{m} / \mathrm{z}$ $742[\mathrm{M}]^{+}$. Anal. calcd for $\mathrm{C}_{32} \mathrm{H}_{30} \mathrm{~N}_{12} \mathrm{O}_{10}: \mathrm{C}, 51.75 ; \mathrm{H}, 4.07 ; \mathrm{N}, 22.63$. Found: $\mathrm{C}, 51.44 ; \mathrm{H}, 3.71 ; \mathrm{N}, 22.34 \%$.

5,5',5",5"'-(((Ethane-1,2-diylbis(oxy))bis-(4,1-phenylene))bis(methanetriyl))tetrakis-(6-aminopyrimidine2,4(1H,3H)-dione) (3b). Yellowish white crystals (623 mg, 84\%), m. p. $>300{ }^{\circ} \mathrm{C} . \mathrm{IR}(\mathrm{KBr}): v_{\max } 3377,3144(\mathrm{br}$, $\mathrm{NH}_{2}$ and $\mathrm{NH}$ ), 1711 (CO), 1624 (CO) cm ${ }^{-1} .{ }^{1} \mathrm{H}$ NMR (400 MHz, DMSO-d 6 ): $\delta 4.23(\mathrm{~s}, 4 \mathrm{H}, 2 \mathrm{OCH}$ ), $5.26(\mathrm{~s}, 2 \mathrm{H}, 2$ $\mathrm{CH}-5$ ), 6.70 (br s, $8 \mathrm{H}, 4 \mathrm{NH}_{2}$ ), 6.81-6.98 (m, $\left.8 \mathrm{H}, \mathrm{Ar}-\mathrm{H}\right), 10.28$ (br s, $\left.2 \mathrm{H}, 2 \mathrm{NH}-1\right), 10.48$ (br s, $\left.2 \mathrm{H}, 4 \mathrm{NH}-3\right) \mathrm{ppm}$. ${ }^{13} \mathrm{C}$ NMR (100 MHz, DMSO-d $): \delta 32.3(\mathrm{CH}), 66.7\left(\mathrm{OCH}_{2}\right), 67.4(\mathrm{CS}), 114.2(\mathrm{Ar}-\mathrm{CH}), 128.1(\mathrm{Ar}-\mathrm{CH}), 131.1(\mathrm{Ar}-\mathrm{C})$, 150.2 (Ar-C), 156.3 and 163.8 (CO-2 and CO-4) ppm. MS (EI, 70 eV): m/z 742 [M] ${ }^{+}$. Anal. calcd for $\mathrm{C}_{32} \mathrm{H}_{30} \mathrm{~N}_{12} \mathrm{O}_{10}$ : C, 51.75; H, 4.07; N, 22.63. Found: C, 51.38; H, 3.87; N, 22.19\%. 
5,5',5",5"'-(((Propane-1,3-diylbis(oxy))bis-(2,1-phenylene))bis(methanetriyl))tetrakis-(6-aminopyrimidine$\mathbf{2 , 4}(\mathbf{1 H}, \mathbf{3 H})$-dione) (3c). Yellowish white crystals (590 mg, 78\%), m. p. 288-290 ${ }^{\circ} \mathrm{C}$. IR ( $\left.\mathrm{KBr}\right): v_{\max } 3377,3185(\mathrm{br}$, $\mathrm{NH}_{2}$ and $\mathrm{NH}$ ), $1712(\mathrm{CO}), 1631$ (CO) cm ${ }^{-1} .{ }^{1} \mathrm{H}$ NMR (400 MHz, DMSO-d $): \delta 2.12\left(\mathrm{~m}, 2 \mathrm{H}, \mathrm{OCH}_{2} \mathrm{CH}_{2}\right), 3.86(\mathrm{~m}, 4 \mathrm{H}$, $2 \mathrm{OCH}_{2} \mathrm{CH}_{2}$ ), 5.30 (s, $\left.2 \mathrm{H}, 2 \mathrm{CH}-5\right), 6.64$ (br s, $\left.8 \mathrm{H}, 4 \mathrm{NH}_{2}\right), 6.77-7.09(\mathrm{~m}, 8 \mathrm{H}, \mathrm{Ar}-\mathrm{H}), 10.11$ (br s, $\left.2 \mathrm{H}, 2 \mathrm{NH}-1\right)$, 10.30 (br s, $2 \mathrm{H}, 4 \mathrm{NH}-3)$ ppm. ${ }^{13} \mathrm{C} \mathrm{NMR}\left(100 \mathrm{MHz}, \mathrm{DMSO}-d_{6}\right): \delta 29.7\left(\mathrm{OCH}_{2} \mathrm{CH}_{2}\right), 32.3(\mathrm{CH}), 63.9\left(\mathrm{OCH}_{2} \mathrm{CH}_{2}\right)$, $65.2(\mathrm{C5}), 113.7(\mathrm{Ar}-\mathrm{CH}), 120.0(\mathrm{Ar}-\mathrm{CH}), 125.1(\mathrm{Ar}-\mathrm{C}), 127.3(\mathrm{Ar}-\mathrm{CH}), 130.3(\mathrm{Ar}-\mathrm{CH}), 150.6(\mathrm{Ar}-\mathrm{C}), 155.6$ and 164.8 (CO-2 and CO-4) ppm, MS (El, $70 \mathrm{eV}$ ): $\mathrm{m} / \mathrm{z} 756[\mathrm{M}]^{+}$. Anal. calcd for $\mathrm{C}_{33} \mathrm{H}_{32} \mathrm{~N}_{12} \mathrm{O}_{10}: \mathrm{C}, 52.38 ; \mathrm{H}, 4.26 ; \mathrm{N}$, 22.21. Found: C, 52.09; $\mathrm{H}, 4.13 ; \mathrm{N}, 22.05 \%$.

5,5',5",5"'-(((Butane-1,4-diylbis(oxy))bis-(2,1-phenylene))bis(methanetriyl))tetrakis-(6-aminopyrimidine-

$\mathbf{2 , 4}(\mathbf{1 H}, \mathbf{3 H})$-dione) (3d). Yellow crystals $(578 \mathrm{mg}, 75 \%), \mathrm{m} . \mathrm{p} .>300{ }^{\circ} \mathrm{C}$. IR $(\mathrm{KBr}): v_{\max } 3358,3180\left(\mathrm{br}, \mathrm{NH}_{2}\right.$ and $\mathrm{NH}), 1708,1631(\mathrm{CO}-2,4) \mathrm{cm}^{-1} .{ }^{1} \mathrm{H}$ NMR $\left(400 \mathrm{MHz}, \mathrm{DMSO}-d_{6}\right): \delta 1.91\left(\mathrm{~m}, 4 \mathrm{H}, 2 \mathrm{OCH}_{2} \mathrm{CH}_{2}\right), 3.90(\mathrm{~m}, 4 \mathrm{H}, 2$ $\mathrm{OCH}_{2} \mathrm{CH}_{2}$ ), 5.29 (s, $\left.2 \mathrm{H}, 2 \mathrm{CH}-5\right), 6.19$ (br s, $8 \mathrm{H}, 4 \mathrm{NH}_{2}$ ), 6.80-7.25 (m, $\left.8 \mathrm{H}, \mathrm{Ar}-\mathrm{H}\right), 10.30$ (br s, $\left.2 \mathrm{H}, 2 \mathrm{NH}-1\right), 10.43$ (br s, $2 \mathrm{H}, 4 \mathrm{NH}-3)$ ppm. ${ }^{13} \mathrm{C} \mathrm{NMR}\left(100 \mathrm{MHz}, \mathrm{DMSO}-d_{6}\right): \delta 29.7\left(\mathrm{OCH}_{2} \mathrm{CH}_{2}\right), 32.5(\mathrm{CH}), 66.8\left(\mathrm{OCH}_{2} \mathrm{CH}_{2}\right), 68.6(\mathrm{C5})$, 113.8 (Ar-CH), $121.0(\mathrm{Ar}-\mathrm{CH}), 125.8(\mathrm{Ar}-\mathrm{CH}), 128.9$ (Ar-C), $129.4(\mathrm{Ar}-\mathrm{CH}), 150.2(\mathrm{Ar}-\mathrm{C}), 155.6$ and 164.6 (CO-2 and CO-4) ppm. MS (El, $70 \mathrm{eV}): m / z 770$ [M] $]^{+}$. Anal. calcd for $\mathrm{C}_{34} \mathrm{H}_{34} \mathrm{~N}_{12} \mathrm{O}_{10}: \mathrm{C}, 52.99 ; \mathrm{H}, 4.45 ; \mathrm{N}, 21.81$. Found: C, $52.74 ; \mathrm{H}, 4.21 ; \mathrm{N}, 21.67 \%$.

5,5',5",5"'-(((Butane-1,4-diylbis(oxy))bis-(4,1-phenylene))bis(methanetriyl))tetrakis-(6-aminopyrimidine2,4(1H,3H)-dione) (3e). Yellowish white crystals (631 mg, 82\%), m. p. 294-296 ${ }^{\circ} \mathrm{C}$. IR (KBr): $v_{\max } 3407,3181$ (br, $\mathrm{NH}$ and $\mathrm{NH}_{2}$ ), 1711 (CO), $1624(\mathrm{CO}) \mathrm{cm}^{-1}$. ${ }^{1} \mathrm{H}$ NMR (400 MHz, DMSO-d 6 ): $\delta 1.84\left(\mathrm{~m}, 4 \mathrm{H}, 2 \mathrm{OCH}_{2} \mathrm{CH}_{2}\right), 3.97(\mathrm{~m}, 4$ $\mathrm{H}, 2 \mathrm{OCH}_{2} \mathrm{CH}_{2}$ ), 5.26 (s, $\left.2 \mathrm{H}, 2 \mathrm{CH}-5\right), 6.64$ (br s, $\left.8 \mathrm{H}, 4 \mathrm{NH}_{2}\right), 6.71-6.97$ (m, $\left.8 \mathrm{H}, \mathrm{Ar}-\mathrm{H}\right), 10.29$ (br s, $\left.2 \mathrm{H}, 2 \mathrm{NH}-1\right)$, 10.48 (br s, $2 \mathrm{H}, 4 \mathrm{NH}-3)$ ppm. ${ }^{13} \mathrm{C} \mathrm{NMR}\left(100 \mathrm{MHz}, \mathrm{DMSO}-d_{6}\right): \delta 26.1\left(\mathrm{OCH}_{2} \mathrm{CH}_{2}\right), 32.0(\mathrm{CH}), 67.5\left(\mathrm{OCH}_{2} \mathrm{CH}_{2}\right)$, $68.2(\mathrm{C5}), 114.1(\mathrm{Ar}-\mathrm{CH}), 127.4(\mathrm{Ar}-\mathrm{CH}), 130.0(\mathrm{Ar}-\mathrm{C}), 150.3(\mathrm{Ar}-\mathrm{C}), 156.7$ and 164.1 (CO-2 and CO-4) ppm. MS (El, $70 \mathrm{eV}): \mathrm{m} / \mathrm{z} 770[\mathrm{M}]^{+}$. Anal. calcd for $\mathrm{C}_{34} \mathrm{H}_{34} \mathrm{~N}_{12} \mathrm{O}_{10}$ : C, 52.99; $\mathrm{H}, 4.45 ; \mathrm{N}, 21.81$. Found: $\mathrm{C}, 52.62 ; \mathrm{H}, 4.19 ; \mathrm{N}$, $21.66 \%$.

5,5',5",5"'-((((1,2-Phenylenebis(methylene))bis(oxy))bis-(2,1-phenylene))bis(methanetriyl))tetrakis-(6aminopyrimidine-2,4(1H,3H)-dione) (3f). Yellowish white crystals $(597 \mathrm{mg}, \mathbf{7 3 \%}), \mathrm{m}$. p. $298-300{ }^{\circ} \mathrm{C} . \mathrm{IR}(\mathrm{KBr})$ : $v_{\max }$ 3368, $3191\left(\mathrm{br}, \mathrm{NH}_{2}\right.$ and $\left.\mathrm{NH}\right), 1706$ (CO), 1631 (CO) $\mathrm{cm}^{-1} .{ }^{1} \mathrm{H}$ NMR (400 MHz, DMSO-d $): \delta 5.02(\mathrm{~s}, 2 \mathrm{H}$, $\mathrm{OCH}_{2}$ ), 5.29 (s, $\left.2 \mathrm{H}, 2 \mathrm{CH}-5\right), 6.62$ (br s, $8 \mathrm{H}, 4 \mathrm{NH}_{2}$ ), 6.83-7.35 (m, $\left.8 \mathrm{H}, \mathrm{Ar}-\mathrm{H}\right), 10.20$ (br s, $\left.2 \mathrm{H}, 2 \mathrm{NH}-1\right), 10.42$ (br s, $2 \mathrm{H}, 4 \mathrm{NH}-3)$ ppm. MS (El, $70 \mathrm{eV}): \mathrm{m} / z 818[\mathrm{M}]^{+}$. Anal. calcd for $\mathrm{C}_{38} \mathrm{H}_{34} \mathrm{~N}_{12} \mathrm{O}_{10}: \mathrm{C}, 55.74 ; \mathrm{H}, 4.19 ; \mathrm{N}, 20.53$. Found: C, 55.42; H, 4.01; N, 20.36\%.

5,5',5",5"'-((((1,4-Phenylenebis(methylene))bis(oxy))bis-(4,1-phenylene))bis(methanetriyl))tetrakis-(6aminopyrimidine-2,4(1H,3H)-dione) $\mathbf{( 3 g )}$. Yellowish white crystals $(622 \mathrm{mg}, 76 \%), \mathrm{m} . \mathrm{p} .>300{ }^{\circ} \mathrm{C} . \mathrm{IR}(\mathrm{KBr}): v_{\max }$ 3410, 3189 (br, NH $\mathrm{NH}_{2}$ and $\mathrm{NH}, 1711$ (CO), 1626 (CO) cm ${ }^{-1} .{ }^{1} \mathrm{H}$ NMR (400 MHz, DMSO-d 6 ): $\delta 5.05\left(\mathrm{~m}, 2 \mathrm{H}, \mathrm{OCH}_{2}\right)$, 5.26 (s, $2 \mathrm{H}, 2 \mathrm{CH}-5), 6.61$ (br s, $8 \mathrm{H}, 4 \mathrm{NH}_{2}$ ), 6.83-7.00 (m, $\left.8 \mathrm{H}, \mathrm{Ar}-\mathrm{H}\right), 10.28$ (br s, 2H, 2NH-1), 10.48 (br s, $2 \mathrm{H}, 4$ $\mathrm{NH}$-3) ppm, ${ }^{13} \mathrm{C}$ NMR (100 MHz, DMSO-d $): \delta 32.2(\mathrm{CH}), 69.3(\mathrm{OCH}), 69.9(\mathrm{C5}), 114.4(\mathrm{Ar}-\mathrm{CH}), 128.2(\mathrm{Ar}-\mathrm{CH})$, $132.3(\mathrm{Ar}-\mathrm{CH}), 136.2(\mathrm{Ar}-\mathrm{C}), 137.4(\mathrm{Ar}-\mathrm{C}), 150.2(\mathrm{Ar}-\mathrm{C}), 155.6$ and 164.8 (CO-2 and CO-4) ppm. MS (El, $70 \mathrm{eV})$ : $\mathrm{m} / \mathrm{z} 818$ [M] $]^{+}$. Anal. calcd for $\mathrm{C}_{38} \mathrm{H}_{34} \mathrm{~N}_{12} \mathrm{O}_{10}: \mathrm{C}, 55.74 ; \mathrm{H}, 4.19 ; \mathrm{N}, 20.53$. Found: $\mathrm{C}, 55.38 ; \mathrm{H}, 3.98 ; \mathrm{N}, 20.42 \%$.

5,5',5",5'"-((((1,3-Phenylenebis(methylene))bis(oxy))bis-(4,1-phenylene))bis(methanetriyl))tetrakis-(6-

aminopyrimidine-2,4(1H,3H)-dione) (3h). Yellowish white crystals (605 mg, 74\%), m. p. 292-294 ${ }^{\circ} \mathrm{C} . \mathrm{IR}(\mathrm{KBr})$ : $v_{\max } 3381,3164\left(\mathrm{br}, \mathrm{NH}_{2}\right.$ and $\mathrm{NH}$ ), 1712 (CO-6), 1627 (CO) cm ${ }^{-1} .{ }^{1} \mathrm{H}$ NMR $\left(400 \mathrm{MHz}, \mathrm{DMSO}-d_{6}\right): \delta 5.06(\mathrm{~s}, 2 \mathrm{H}$, $\mathrm{OCH}_{2}$ ), 5.26 (s, $\left.2 \mathrm{H}, 2 \mathrm{CH}-5\right), 6.61$ (br s, $8 \mathrm{H}, 4 \mathrm{NH}_{2}$ ), 6.85-7.02 (m, $\left.8 \mathrm{H}, \mathrm{Ar}-\mathrm{H}\right), 10.20$ (br s, $\left.2 \mathrm{H}, 2 \mathrm{NH}-1\right), 10.42$ (br s, $2 \mathrm{H}, 4 \mathrm{NH}-3)$ ppm. ${ }^{13} \mathrm{C}$ NMR (100 MHz, DMSO-d $): \delta 32.2(\mathrm{CH}), 69.5\left(\mathrm{OCH}_{2}\right), 70.0(\mathrm{C} 5), 114.4(\mathrm{Ar}-\mathrm{CH}), 127.5$ $(\mathrm{Ar}-\mathrm{CH}), 128.0(\mathrm{Ar}-\mathrm{CH}), 129.1(\mathrm{Ar}-\mathrm{CH}), 132.3(\mathrm{Ar}-\mathrm{CH}), 136.2(\mathrm{Ar}-\mathrm{C}), 138.0(\mathrm{Ar}-\mathrm{C}), 150.2(\mathrm{Ar}-\mathrm{C}), 155.6$ and 164.7 
(CO-2 and CO-4) ppm. MS (El, 70 eV): m/z 818 [M] $]^{+}$. Anal. calcd for $\mathrm{C}_{38} \mathrm{H}_{34} \mathrm{~N}_{12} \mathrm{O}_{10}: \mathrm{C}, 55.74 ; \mathrm{H}, 4.19 ; \mathrm{N}, 20.53$. Found: C, 55.56; H, 4.43; N, 20.18\%.

\section{Synthesis of compounds $9 \mathrm{a}-\mathrm{d}$ and $9 \mathrm{~g}-\mathrm{I}$. General procedure}

A mixture of bisaldehyde 1a-i (1 mmol), 6-aminouracil 2 (254 mg, 2 mmol) and dimedone 7 (280 mg, $2 \mathrm{mmol}$ ) was heated at reflux in glacial $\mathrm{AcOH}(20 \mathrm{~mL})$ for $5 \mathrm{~h}$. The solvent was evaporated under reduced pressure. The residue was treated with aq. $\mathrm{NaHCO}_{3}$ solution $(0.5 \mathrm{~N}, 20 \mathrm{~mL})$, washed thoroughly with distilled $\mathrm{H}_{2} \mathrm{O}(20 \mathrm{~mL})$ and left to dry. The crude product was purified by crystallization from EtOH/1,4-dioxane (1:1, v/v, $10 \mathrm{~mL})$.

5,5'-((Ethane-1,2-diylbis(oxy))bis-(2,1-phenylene))bis-(8,8-dimethyl-5,8,9,10-tetrahydropyrimido[4,5-b]-

quinoline-2,4,6(1H,3H,7H)-trione) (9a). Yellowish white crystals $(608 \mathrm{mg}, \mathbf{8 3 \%}), \mathrm{m}$. p. $292-294^{\circ} \mathrm{C} . \mathrm{IR}(\mathrm{KBr}): \boldsymbol{v}_{\max }$ $3424(\mathrm{NH}), 3294(\mathrm{NH}), 3227(\mathrm{NH}), 1721(\mathrm{CO}-6), 1632$ (br, CO-2,4) cm-1. ${ }^{1} \mathrm{H}$ NMR (400 MHz, DMSO-d $)$ : single stereoisomer, $\delta 0.80\left(\mathrm{~s}, 6 \mathrm{H}, 2 \mathrm{CH}_{3}\right), 0.99\left(\mathrm{~s}, 6 \mathrm{H}, 2 \mathrm{CH}_{3}\right), 1.90-2.29\left(\mathrm{~m}, 8 \mathrm{H}, 2 \mathrm{CH}_{2}-9\right.$ and $\left.2 \mathrm{CH}_{2}-7\right), 4.10-4.25(\mathrm{~m}$, $\left.4 \mathrm{H}, 2 \mathrm{OCH}_{2}\right), 4.86(\mathrm{~s}, 2 \mathrm{H}, 2 \mathrm{CH}-5), 6.81-7.27$ (m, $\left.8 \mathrm{H}, \mathrm{Ar}-\mathrm{H}\right), 8.98$ (br s, $\left.2 \mathrm{H}, 2 \mathrm{NH}-10\right), 10.11$ (br s, $\left.2 \mathrm{H}, 2 \mathrm{NH}-1\right)$, 10.56 (br s, $2 \mathrm{H}, 2 \mathrm{NH}-3)$ ppm. ${ }^{13} \mathrm{C}$ NMR (100 MHz, DMSO-d $): \delta 26.5\left(\mathrm{CH}_{3}\right), 29.7\left(\mathrm{CH}_{3}\right), 32.5(\mathrm{C}-8), 33.0(\mathrm{CH})$, $40.6\left(\mathrm{CH}_{2}-9\right), 50.7\left(\mathrm{CH}_{2}-7\right), 66.2\left(\mathrm{OCH}_{2}\right), 88.6(\mathrm{C}-4 \mathrm{a}), 110.1(\mathrm{C}-5 \mathrm{a}), 112.7(\mathrm{Ar}-\mathrm{CH}), 120.3(\mathrm{Ar}-\mathrm{CH}), 127.7(\mathrm{Ar}-\mathrm{CH})$, 132.7 (Ar-CH), $133.2(\mathrm{Ar}-\mathrm{C}), 149.7$ (C-9a), $150.4(\mathrm{Ar}-\mathrm{C}), 157.2$ and 163.1 (CO-2, CO-4), 194.6 (CO-6) ppm. MS (El, $70 \mathrm{eV}): m / z 732$ [M] $]^{+}$. Anal. calcd for $\mathrm{C}_{40} \mathrm{H}_{40} \mathrm{~N}_{6} \mathrm{O}_{8}: \mathrm{C}, 65.56 ; \mathrm{H}, 5.50 ; \mathrm{N}, 11.47$. Found: $\mathrm{C}, 65.29 ; \mathrm{H}, 5.31 ; \mathrm{N}$, $11.17 \%$.

5,5'-((Ethane-1,2-diylbis(oxy))bis-(4,1-phenylene))bis-(8,8-dimethyl-5,8,9,10-tetrahydropyrimido[4,5-b]quinoline-2,4,6(1H,3H,7H)-trione) (9b). Yellow crystals (622 mg, 85\%), m. p. 294-296 ${ }^{\circ} \mathrm{C}$. IR $(\mathrm{KBr}): V_{\max } 3424$ (NH), $3286(\mathrm{NH}), 3222(\mathrm{NH}), 1719$ (CO-6), 1664 (br, CO-2,4) cm-1. ${ }^{1} \mathrm{H}$ NMR (400 MHz, DMSO-d 6$): \delta 0.89(\mathrm{~s}, 6 \mathrm{H}, 2$ $\left.\mathrm{CH}_{3}\right), 1.02\left(\mathrm{~s}, 6 \mathrm{H}, 2 \mathrm{CH}_{3}\right), 1.92-2.22\left(\mathrm{~m}, 4 \mathrm{H}, 2 \mathrm{CH}_{2}-9\right), 2.39-2.45\left(\mathrm{~m}, 4 \mathrm{H}, 2 \mathrm{CH}_{2}-7\right), 4.18\left(\mathrm{~s}, 4 \mathrm{H}, 2 \mathrm{OCH}_{2}\right), 4.69(\mathrm{~s}$, $2 \mathrm{H}, 2 \mathrm{CH}-5), 6.77-7.10$ (m, $8 \mathrm{H}, \mathrm{Ar}-\mathrm{H}), 8.79$ (br s, $2 \mathrm{H}, 2 \mathrm{NH}-10), 10.12$ (br s, $2 \mathrm{H}, 2 \mathrm{NH}-1), 10.70$ (br s, $2 \mathrm{H}, 2 \mathrm{NH}-$ 3) ppm. ${ }^{13} \mathrm{C}$ NMR (100 MHz, DMSO-d $): \delta 27.0\left(\mathrm{CH}_{3}\right), 29.4\left(\mathrm{CH}_{3}\right), 32.6(C-8), 32.7(\mathrm{CH}), 40.6\left(\mathrm{CH}_{2}-9\right), 50.6\left(\mathrm{CH}_{2^{-}}\right.$ 7), $66.7\left(\mathrm{OCH}_{2}\right), 90.3$ (C-4a), 111.9 (C-5a), 114.3 (Ar-CH), $129.0(\mathrm{Ar}-\mathrm{CH}), 139.5$ (Ar-C), 149.3 (C-9a), 156.9 and 163.2 (CO-2 and CO-4), 194.8 (CO-6) ppm. MS (El, $70 \mathrm{eV}): \mathrm{m} / z 732$ [M] . Anal. calcd for $\mathrm{C}_{40} \mathrm{H}_{40} \mathrm{~N}_{6} \mathrm{O}_{8}: \mathrm{C}, 65.56 ; \mathrm{H}$, 5.50; N, 11.47. Found: C, 65.34; H, 5.27; N, 11.21\%

5,5'-((Propane-1,3-diylbis(oxy))bis-(2,1-phenylene))bis-(8,8-dimethyl-5,8,9,10-tetrahydropyrimido[4,5-b]quinoline-2,4,6(1H,3H,7H)-trione) (9c). Yellow crystals (642 mg, 86\%), m. p. 266-268 ${ }^{\circ} \mathrm{C}$. IR (KBr): $v_{\max } 3411$ $(\mathrm{NH}), 3265(\mathrm{NH}), 3159(\mathrm{NH}), 1708$ (CO-6), 1638 (br, CO-2,4) cm-1. ${ }^{1} \mathrm{H}$ NMR (400 MHz, DMSO-d $)$ : major diastereomer, $\delta 0.80\left(\mathrm{~s}, 6 \mathrm{H}, 2 \mathrm{CH}_{3}\right), 0.98\left(\mathrm{~s}, 6 \mathrm{H}, 2 \mathrm{CH}_{3}\right), 1.91\left(\mathrm{~m}, 2 \mathrm{H}, \mathrm{OCH}_{2} \mathrm{CH}_{2}\right), 2.12-2.47\left(\mathrm{~m}, 8 \mathrm{H}, 2 \mathrm{CH}_{2}-9\right.$ and $\left.2 \mathrm{CH}_{2}-7\right), 4.01\left(\mathrm{~m}, 4 \mathrm{H}, 2 \mathrm{OCH}_{2} \mathrm{CH}_{2}\right), 4.90(\mathrm{~s}, 2 \mathrm{H}, 2 \mathrm{CH}-5), 6.72-7.24(\mathrm{~m}, 8 \mathrm{H}, \mathrm{Ar}-\mathrm{H}), 8.75(\mathrm{br} \mathrm{s}, 2 \mathrm{H}, 2 \mathrm{NH}-10)$, 10.12 (br s, $2 \mathrm{H}, 2 \mathrm{NH}-1$ ), 10.60 (br s, $2 \mathrm{H}, 2 \mathrm{NH}-3$ ) ppm. ${ }^{13} \mathrm{C} \mathrm{NMR}\left(100 \mathrm{MHz}, \mathrm{DMSO}-d_{6}\right): \delta 26.4\left(\mathrm{CH}_{3}\right), 29.6$ $\left(\mathrm{OCH}_{2} \mathrm{CH}_{2}\right), 29.9\left(\mathrm{CH}_{3}\right), 32.5(\mathrm{C}-8), 32.8(\mathrm{CH}), 40.6\left(\mathrm{CH}_{2}-9\right), 50.8\left(\mathrm{CH}_{2}-7\right), 65.1\left(\mathrm{OCH}_{2} \mathrm{CH}_{2}\right), 89.1(\mathrm{C}-4 \mathrm{a}), 110.5(\mathrm{C}-$ 5a), $111.9(\mathrm{Ar}-\mathrm{CH}), 119.8(\mathrm{Ar}-\mathrm{CH}), 127.6(\mathrm{Ar}-\mathrm{CH}), 132.3(\mathrm{Ar}-\mathrm{CH}), 133.4(\mathrm{Ar}-\mathrm{C}), 149.4$ (C-9a), $152.5(\mathrm{Ar}-\mathrm{C}), 157.4$ and 163.1 (CO-2), 163.2 (CO-4), 194.5 (CO-6) ppm. MS (El, 70 eV): m/z 746 [M] $]^{+}$Anal. calcd for $\mathrm{C}_{41} \mathrm{H}_{42} \mathrm{~N}_{6} \mathrm{O}_{8}: \mathrm{C}_{\text {, }}$ $65.94 ; \mathrm{H}, 5.67 ; \mathrm{N}, 11.25$. Found: C, 65.63; H, 5.41; N, $11.09 \%$

5,5'-((Butane-1,4-diylbis(oxy))bis-(2,1-phenylene))bis-(8,8-dimethyl-5,8,9,10-tetrahydropyrimido[4,5-b]quinoline-2,4,6(1H,3H,7H)-trione) (9d). Yellowish white crystals $(669 \mathrm{mg}, 88 \%), \mathrm{m}$. p. $>300{ }^{\circ} \mathrm{C} . \mathrm{IR}(\mathrm{KBr}): \boldsymbol{v}_{\max }$ 3431, $3291(\mathrm{br}, 3 \mathrm{NH}), 1714$ (CO-6), 1647 (CO-2 and CO-4) cm-1. ${ }^{1} \mathrm{H}$ NMR (400 MHz, DMSO-d $d_{6}$ : a pair of diastereomers (1:1), $\delta 0.83(0.84)\left(\mathrm{s}, 6 \mathrm{H}, 2 \mathrm{CH}_{3}\right), 0.97(0.99)\left(\mathrm{s}, 6 \mathrm{H}, 2 \mathrm{CH}_{3}\right), 1.85\left(\mathrm{~m}, 4 \mathrm{H}, 2 \mathrm{OCH}_{2} \mathrm{CH}_{2}\right), 2.12-2.48$ ( $\mathrm{m}, 8 \mathrm{H}, 2 \mathrm{CH}_{2}-9$ and $\left.2 \mathrm{CH}_{2}-7\right), 3.89(4.00)\left(\mathrm{m}, 4 \mathrm{H}, 2 \mathrm{OCH}_{2} \mathrm{CH}_{2}\right), 4.89(4.96)(\mathrm{s}, 2 \mathrm{H}, 2 \mathrm{CH}-5), 6.72-7.24(\mathrm{~m}, 8 \mathrm{H}$, Ar-H), 8.68 (8.73) (br s, $2 \mathrm{H}, 2 \mathrm{NH}-10), 10.06$ (10.10) (br s, $2 \mathrm{H}, 2 \mathrm{NH}-1), 10.59$ (10.67) (br s, $2 \mathrm{H}, 2 \mathrm{NH}-3) \mathrm{ppm}$. ${ }^{13} \mathrm{C}$ NMR (100 MHz, DMSO-d $\left.d_{6}\right)$ : a pair of diastereomers $(1: 1), \delta 26.2(26.4)\left(\mathrm{CH}_{3}\right), 26.6(26.7)\left(\mathrm{OCH}_{2} \mathrm{CH}_{2}\right), 29.6$ (29.7) $\left(\mathrm{CH}_{3}\right), 32.4(32.5)(\mathrm{C}-8), 32.6(32.7)(\mathrm{CH}), 40.6(40.7)\left(\mathrm{CH}_{2}-9\right), 50.7(50.8)\left(\mathrm{CH}_{2}-7\right), 68.0(68.1)\left(\mathrm{OCH}_{2} \mathrm{CH}_{2}\right)$, 
89.0 (89.4) (C-4a), 110.4 (110.8) (C-5a), 112.0 (112.4) (Ar-CH), 119.6 (119.8) (Ar-CH), 127.5 (127.6) (Ar-CH), 132.0 (132.5) (Ar-CH), 133.3 (133.7) (Ar-C), 149.5 (149.7) (C-9a), 150.3 (150.4) (C-10a), 157.3 (157.6) and 163.1 (163.4) (CO-2 and CO-4), 194.5 (194.7) (CO-6) ppm. MS (El, 70 eV): m/z 760 [M] $]^{+}$. Anal. calcd for $\mathrm{C}_{42} \mathrm{H}_{44} \mathrm{~N}_{6} \mathrm{O}_{8}: \mathrm{C}_{\text {, }}$ 66.30; H, 5.83; N, 11.05. Found: C, 66.09; H, 5.44; N, 10.78\%.

5,5'-(((1,4-Phenylenebis(methylene))bis(oxy))bis-(4,1-phenylene))bis-(8,8-dimethyl-5,8,9,10-tetrahydropyrimido[4,5-b]quinoline-2,4,6(1H,3H,7H)-trione) $(\mathbf{9 g})$. Pale yellow crystals $(719 \mathrm{mg}, 89 \%), \mathrm{m}$. p. $>300{ }^{\circ} \mathrm{C}$. IR $(\mathrm{KBr}): v_{\max } 3428,3198(\mathrm{br}, \mathrm{NH}), 1720$ (CO-6), 1664 (br, CO-2 and CO-4) cm ${ }^{-1} .{ }^{1} \mathrm{H}$ NMR (400 MHz, DMSO-d 6 ): single stereoisomer, $\delta 0.90\left(\mathrm{~s}, 6 \mathrm{H}, 2 \mathrm{CH}_{3}\right), 1.02\left(\mathrm{~s}, 6 \mathrm{H}, 2 \mathrm{CH}_{3}\right), 2.00-2.21\left(\mathrm{~m}, 4 \mathrm{H}, 2 \mathrm{CH}_{2}-9\right), 2.43\left(\mathrm{~m}, 4 \mathrm{H}, 2 \mathrm{CH}_{2-}\right.$ 7), $4.70(\mathrm{~s}, 2 \mathrm{H}, 2 \mathrm{CH}-5), 5.01\left(\mathrm{~s}, 4 \mathrm{H}, 2 \mathrm{OCH}_{2}\right), 6.81-7.10(\mathrm{~m}, 8 \mathrm{H}, \mathrm{Ar}-\mathrm{H}-4,1-$ phenylene), 7.41 (s, 4H, Ar-H-1,4phenylene), 8.79 (br s, 2H, 2NH-10), 10.27 (br s, 2H, 2NH-1), 10.70 (br s, 2H, 2NH-3) ppm. ${ }^{13} \mathrm{C} \mathrm{NMR}(100 \mathrm{MHz}$, DMSO-d $\left.d_{6}\right): \delta 27.0\left(\mathrm{CH}_{3}\right), 29.4\left(\mathrm{CH}_{3}\right), 32.4(\mathrm{C}-8), 32.6(\mathrm{CH}), 40.6\left(\mathrm{CH}_{2}-9\right), 50.6\left(\mathrm{CH}_{2}-7\right), 69.3\left(\mathrm{OCH}_{2}\right), 90.3(\mathrm{C}-4 \mathrm{a})$, 111.9 (C-5a), 114.5 (Ar-CH), $128.1(\mathrm{Ar}-\mathrm{CH}), 129.0(\mathrm{Ar}-\mathrm{CH}), 137.3(\mathrm{Ar}-\mathrm{C}), 139.5$ (Ar-C), $149.4(\mathrm{C}-9 \mathrm{a}), 150.2$ (C10a), 156.7 and 163.2 (CO-2 and CO-4), 194.6 (CO-6) ppm. MS (El, $70 \mathrm{eV}): \mathrm{m} / \mathrm{z} 808$ [M] . Anal. calcd for $\mathrm{C}_{46} \mathrm{H}_{44} \mathrm{~N}_{6} \mathrm{O}_{8}$ : C, 68.30; $\mathrm{H}, 5.48 ; \mathrm{N}, 10.39$. Found: C, 68.13; $\mathrm{H}, 5.12 ; \mathrm{N}, 10.13 \%$.

5,5'-(((1,3-Phenylenebis(methylene))bis(oxy))bis-(4,1-phenylene))bis-(8,8-dimethyl-5,8,9,10-tetrahydropyrimido[4,5-b]quinoline-2,4,6(1H,3H,7H)-trione) (9h). Yellow crystals (663 mg, 82\%), m. p. $>300{ }^{\circ} \mathrm{C} . \mathrm{IR}(\mathrm{KBr})$ : $v_{\max } 3205$ (br, NH), 1717 (CO-6), 1664 (CO-2 and CO-4) cm ${ }^{-1} .{ }^{1} \mathrm{H}$ NMR (400 MHz, DMSO-d 6 ): $\delta 0.90(\mathrm{~s}, 6 \mathrm{H}, 2$ $\left.\mathrm{CH}_{3}\right), 1.02\left(\mathrm{~s}, 6 \mathrm{H}, 2 \mathrm{CH}_{3}\right), 2.00-2.22\left(\mathrm{~m}, 4 \mathrm{H}, 2 \mathrm{CH}_{2}-9\right), 2.44\left(\mathrm{~m}, 4 \mathrm{H}, 2 \mathrm{CH}_{2}-7\right), 4.70(\mathrm{~s}, 2 \mathrm{H}, 2 \mathrm{CH}-5), 5.02(\mathrm{~s}, 4 \mathrm{H}, 2$ $\mathrm{OCH}_{2}$ ), 6.82-7.10 (m, $8 \mathrm{H}, \mathrm{Ar}-\mathrm{H}-4,1-$ phenylene), 7.37 (s, $3 \mathrm{H}, \mathrm{Ar}-\mathrm{H}-$, 4,5,6-1,3-phenylene), 7.47 (s, $1 \mathrm{H}, \mathrm{Ar}-\mathrm{H} 2-$ 1,3-phenylene), 8.80 (br s, $2 \mathrm{H}, 2 \mathrm{NH}-10), 10.27$ (br s, $2 \mathrm{H}, 2 \mathrm{NH}-1), 10.70$ (br s, $2 \mathrm{H}, 2 \mathrm{NH}-3)$ ppm. ${ }^{13} \mathrm{C} \mathrm{NMR}(100$ $\left.\mathrm{MHz}, \mathrm{DMSO}-d_{6}\right): \delta 27.0\left(\mathrm{CH}_{3}\right), 29.4\left(\mathrm{CH}_{3}\right), 32.5(\mathrm{C}-8), 32.6(\mathrm{CH}), 40.6\left(\mathrm{CH}_{2}-9\right), 50.6\left(\mathrm{CH}_{2}-7\right), 69.5\left(\mathrm{OCH}_{2}\right), 90.3(C-$ 4a), 111.9 (C-5a), $114.4(\mathrm{Ar}-\mathrm{CH}), 127.5(\mathrm{Ar}-\mathrm{CH}), 127.5$ (Ar-CH), $128.9(\mathrm{Ar}-\mathrm{CH}), 137.9(\mathrm{Ar}-\mathrm{C}), 139.5(\mathrm{Ar}-\mathrm{C}), 149.4$ (C-9a), 150.3 (C-10a), 157.0 and 163.2 (CO-2 and CO-4), 194.8 (CO-6) ppm. MS (El, 70 eV): m/z 808 [M] ${ }^{+}$. Anal. calcd for $\mathrm{C}_{46} \mathrm{H}_{44} \mathrm{~N}_{6} \mathrm{O}_{8}$ : C, 68.30; $\mathrm{H}, 5.48 ; \mathrm{N}, 10.39$. Found: $\mathrm{C}, 68.03 ; \mathrm{H}, 5.19 ; \mathrm{N}, 10.17 \%$.

5,5'-(((1,4-Phenylenebis(methylene))bis(oxy))bis-(2,1-phenylene))bis-(8,8-dimethyl-5,8,9,10-tetrahydropyrimido[4,5-b]quinoline-2,4,6(1H,3H,7H)-trione) (9i). Yellowish white crystals $(703 \mathrm{mg}, 87 \%), \mathrm{m} . \mathrm{p} .>300{ }^{\circ} \mathrm{C}$. IR (KBr): $v_{\max } 3416,3234(\mathrm{br}, \mathrm{NH}), 1712$ (CO-6), 1650 (CO-2 and CO-4) cm ${ }^{-1},{ }^{1} \mathrm{H}$ NMR (400 MHz, DMSO-d $): \delta$ $0.85\left(\mathrm{~s}, 6 \mathrm{H}, 2 \mathrm{CH}_{3}\right), 0.97\left(\mathrm{~s}, 6 \mathrm{H}, 2 \mathrm{CH}_{3}\right), 1.91-2.37\left(\mathrm{~m}, 8 \mathrm{H}, 2 \mathrm{CH}_{2}-9\right.$ and $\left.2 \mathrm{CH}_{2}-7\right), 5.00(\mathrm{~s}, 2 \mathrm{H}, 2 \mathrm{CH}-5), 5.08(\mathrm{~s}, 4$ $\mathrm{H}, 2 \mathrm{OCH}_{2}$ ), 6.75 -7.19 (m, $8 \mathrm{H}, \mathrm{Ar}-\mathrm{H}-2,1$-phenylene), 7.44 (s, 4H, Ar-H-1,4-phenylene), 8.33 (br s, $\left.2 \mathrm{H}, 2 \mathrm{NH}-10\right)$, 10.06 (br s, $2 \mathrm{H}, 2 \mathrm{NH}-1), 10.58$ (br s, $2 \mathrm{H}, 2 \mathrm{NH}-3)$ ppm. ${ }^{13} \mathrm{C} \mathrm{NMR}(100 \mathrm{MHz}$, DMSO-d 6$): \delta 26.9\left(\mathrm{CH}_{3}\right), 29.4\left(\mathrm{CH}_{3}\right)$, $32.4(\mathrm{C}-8), 32.7(\mathrm{CH}), 40.6\left(\mathrm{CH}_{2}-9\right), 50.8\left(\mathrm{CH}_{2}-7\right), 69.6\left(\mathrm{OCH}_{2}\right), 89.5(\mathrm{C}-4 \mathrm{a}), 110.8(\mathrm{C}-5 \mathrm{a}), 112.6(\mathrm{Ar}-\mathrm{CH}), 120.1(\mathrm{Ar}-$ $\mathrm{CH}), 127.4(\mathrm{Ar}-\mathrm{CH}), 127.8(\mathrm{Ar}-\mathrm{CH}), 131.6(\mathrm{Ar}-\mathrm{CH}), 137.6(\mathrm{Ar}-\mathrm{C}), 144.4(\mathrm{Ar}-\mathrm{C}), 149.5$ (C-9a), 150.3 (C-10a), 156.7 and 163.0 (CO-2 and CO-4), 194.5 (CO-6) ppm. MS (El, $70 \mathrm{eV}): \mathrm{m} / z 808$ [M] ${ }^{+}$. Anal. calcd for $\mathrm{C}_{46} \mathrm{H}_{44} \mathrm{~N}_{6} \mathrm{O}_{8}: \mathrm{C}_{\text {, }}$ 68.30; H, 5.48; N, 10.39. Found: C, 68.08; H, 5.16; N, 10.21\%.

\section{Synthesis of compounds $15 \mathrm{c}, 15 \mathrm{~d}, 15 \mathrm{~h}$ and $15 \mathrm{i}$. General procedure}

A mixture of bisaldehyde 1c, $1 \mathbf{d}, \mathbf{1 h}$ or $\mathbf{1 i}(1 \mathrm{mmol}), 6$-aminouracil $\mathbf{2}$ (254 mg, $2 \mathrm{mmol}$ ) and 1,3-indanedione 13 (292 mg, $2 \mathrm{mmol}$ ) was heated at reflux in glacial AcOH $(20 \mathrm{~mL})$ for $5 \mathrm{~h}$. The solvent was evaporated under reduced pressure. The residue was treated with aq. $\mathrm{NaHCO}_{3}$ solution $(0.5 \mathrm{~N}, 20 \mathrm{~mL})$, washed thoroughly with distilled $\mathrm{H}_{2} \mathrm{O}(20 \mathrm{~mL})$ and left to dry. The crude product was purified by crystallization from EtOH/1,4-dioxane $(1: 1, v / v, 10 \mathrm{~mL})$.

5,5'-((Propane-1,3-diylbis(oxy))bis-(2,1-phenylene))bis-(5,11-dihydro-1H-indeno[2',1':5,6]pyrido[2,3-d]pyrimidine-2,4,6(3H)-trione) (15c). Orange crystals (584 mg, 77\%), m. p. $>300{ }^{\circ} \mathrm{C}$. IR (KBr): $v_{\max } 3430,3276(\mathrm{br}$, $\mathrm{NH}), 1725$ (CO-6), 1648, $1612(\mathrm{CO}-2,4) \mathrm{cm}^{-1} .{ }^{1} \mathrm{H}$ NMR $(400 \mathrm{MHz}$, DMSO-d $)$ : two diastereomers (1:1) $\delta 2.08(\mathrm{br}$ $\left.\mathrm{m}, 2 \mathrm{H}, 2 \mathrm{OCH}_{2} \mathrm{CH}_{2}\right), 3.74$ (3.87) (m, $\left.4 \mathrm{H}, 2 \mathrm{OCH}_{2} \mathrm{CH}_{2}\right), 4.76(4.83)(\mathrm{s}, 2 \mathrm{H}, 2 \mathrm{CH}-5), 6.10-7.52(\mathrm{~m}, 16 \mathrm{H}, \mathrm{Ar}-\mathrm{H})$, 
10.07 (br s, $2 \mathrm{H}, 2 \mathrm{NH}-11), 10.18$ (10.25) (br s, $2 \mathrm{H}, 2 \mathrm{NH}-1), 10.78$ (10.82) (br s, $2 \mathrm{H}, 2 \mathrm{NH}-3)$ ppm. ${ }^{13} \mathrm{C} \mathrm{NMR}(100$ $\left.\mathrm{MHz}, \mathrm{DMSO}-d_{6}\right)$ : two diastereomers, $\delta 29.8(29.9)\left(\mathrm{OCH}_{2} \mathrm{CH}_{2}\right), 31.7$ (32.3) $(\mathrm{CH}), 64.6(64.8)\left(\mathrm{OCH}_{2}\right), 91.0(91.2)$ (C-4a), 109.2 (109.4) (C-5a), 111.5 (111.8) (Ar-CH), 119.0 (119.1) (Ar-CH), 119.9 (120.1) (Ar-CH), 121.1 (121.2) (Ar-CH), 127.9 (128.0) (Ar-CH), 130.6 (130.7) (Ar-CH), 131.5 (131.7) (Ar-CH), 131.8 (132.2) (Ar-C), 132.4 (132.5) (Ar-CH), 133.2 (133.3) (Ar-C), 136.3 (136.4) (Ar-C), 145.3 (150.3) (C-10b), 154.3 (154.4) (C-11a), 157.3 (157.4) and 163.1 (163.2) (CO-2 and CO-4), 191.1 (191.2) (CO-6) ppm. MS (El, $70 \mathrm{eV}): \mathrm{m} / \mathrm{z} 758$ [M] ${ }^{+}$. Anal. calcd for $\mathrm{C}_{43} \mathrm{H}_{30} \mathrm{~N}_{6} \mathrm{O}_{8}: \mathrm{C}, 68.07 ; \mathrm{H}, 3.99 ; \mathrm{N}, 11.08$. Found: $\mathrm{C}, 67.81 ; \mathrm{H}, 3.73 ; \mathrm{N}, 10.77 \%$.

5,5'-((Butane-1,4-diylbis(oxy))bis-(2,1-phenylene))bis-(5,11-dihydro-1H-indeno[2',1':5,6]pyrido[2,3-d]-

pyrimidine-2,4,6(3H)-trione) (15d). Orange crystals (648 mg, 84\%), m. p. 290-292 ${ }^{\circ} \mathrm{C} . \mathrm{IR}(\mathrm{KBr}): v_{\max } 3427(\mathrm{br}$, $\mathrm{NH}), 1713$ (CO-6), 1655 (CO-2 and CO-4) cm ${ }^{-1} .{ }^{1} \mathrm{H}$ NMR (400 MHz, DMSO-d ${ }_{6}$ ): major diastereomer, $\delta 1.72(\mathrm{~m}, 4$ $\left.\mathrm{H}, 2 \mathrm{OCH}_{2} \mathrm{CH}_{2}\right), 3.89\left(\mathrm{~m}, 4 \mathrm{H}, \mathrm{OCH}_{2} \mathrm{CH}_{2}\right), 4.87(\mathrm{~s}, 2 \mathrm{H}, 2 \mathrm{CH}-5), 6.79-7.43(\mathrm{~m}, 16 \mathrm{H}, \mathrm{Ar}-\mathrm{H}), 10.08(\mathrm{br} \mathrm{s}, 2 \mathrm{H}, 2 \mathrm{NH}-$

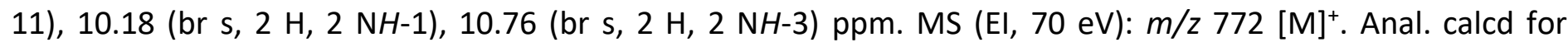
$\mathrm{C}_{44} \mathrm{H}_{32} \mathrm{~N}_{6} \mathrm{O}_{8}: \mathrm{C}, 68.39 ; \mathrm{H}, 4.17 ; \mathrm{N}, 10.88$. Found: $\mathrm{C}, 68.13 ; \mathrm{H}, 4.04 ; \mathrm{N}, 10.69$.

5,5'-(((1,3-Phenylenebis(methylene))bis(oxy))bis-(4,1-phenylene))bis-(5,11-dihydro-1H-indeno[2',1':5,6]pyrido[2,3-d]pyrimidine-2,4,6(3H)-trione) (15h). Brick red crystals (631 mg, 77\%), m. p. 296-298 ${ }^{\circ} \mathrm{C} . \mathrm{IR}(\mathrm{KBr})$ : $v_{\max }$ 3420, $3244\left(\mathrm{br}, \mathrm{NH}_{2}\right.$ and $\left.\mathrm{NH}\right), 1712$ (CO-6), 1608 (CO-2 and CO-4) cm ${ }^{-1} .{ }^{1} \mathrm{H}$ NMR (400 MHz, DMSO-d $\left.d_{6}\right): \delta$ 4.62 (s, $2 \mathrm{H}, 2 \mathrm{CH}-5), 5.02\left(\mathrm{~s}, 4 \mathrm{H}, 2 \mathrm{CH}_{2}\right), 6.85-7.42(\mathrm{~m}, 20 \mathrm{H}, \mathrm{Ar}-\mathrm{H}), 10.14$ (br s, $\left.2 \mathrm{H}, 2 \mathrm{NH}-11\right), 10.31$ (br s, $2 \mathrm{H}, 2$ $\mathrm{NH}-1), 10.90$ (br s, $2 \mathrm{H}, 2 \mathrm{NH}-3)$ ppm. ${ }^{13} \mathrm{C}$ NMR (100 MHz, DMSO-d $): \delta 33.1(\mathrm{CH}), 69.5\left(\mathrm{OCH}_{2} \mathrm{CH}_{2}\right), 91.7(\mathrm{C}-4 \mathrm{a})$, 110.3 (C-5a), 114.7 (Ar-CH), $119.4(\mathrm{Ar}-\mathrm{CH}), 121.3(\mathrm{Ar}-\mathrm{CH}), 127.4(\mathrm{Ar}-\mathrm{CH}), 127.5(\mathrm{Ar}-\mathrm{CH}), 129.1(\mathrm{Ar}-\mathrm{CH}), 130.8$ (Ar-CH), 132.6 (Ar-CH), $133.1(\mathrm{Ar}-\mathrm{C}), 136.4(\mathrm{Ar}-\mathrm{C}), 137.5$ (Ar-CH), 137.9 (Ar-C), 138.2 (Ar-C), 144.9 (C-10b), 153.7 (C-11a), 157.3 and 163.3 (CO-2 and CO-4), 191.4 (CO-6) ppm. MS (El, $70 \mathrm{eV}): \mathrm{m} / \mathrm{z} 820$ [M] $^{+}$. Anal. calcd for $\mathrm{C}_{48} \mathrm{H}_{32} \mathrm{~N}_{6} \mathrm{O}_{8}: \mathrm{C}, 70.24 ; \mathrm{H}, 3.93 ; \mathrm{N}, 10.24$. Found: C, 70.01; $\mathrm{H}, 3.66 ; \mathrm{N}, 10.06 \%$.

5,5'-(((1,4-Phenylenebis(methylene))bis(oxy))bis-(4,1-phenylene))bis-(5,11-dihydro-1H-indeno[2',1':5,6]pyrido[2,3-d]pyrimidine-2,4,6(3H)-trione) (15i). Pale brown crystals (705 mg,86\%), m. p. $>300{ }^{\circ} \mathrm{C} . \mathrm{IR}(\mathrm{KBr}): v_{\max }$ 3427, 3168 (br, NH 2 and NH), 1714 (CO-6), 1659, 1608 (CO-2 and CO-4) cm-1 . ${ }^{1} \mathrm{H}$ NMR (400 MHz, DMSO-d $\left.d_{6}\right): \delta$ 4.97-5.14 (m, $6 \mathrm{H}, 2 \mathrm{CH}-5$ and $\left.2 \mathrm{CH}_{2}\right), 6.81-7.83(\mathrm{~m}, 20 \mathrm{H}, \mathrm{Ar}-\mathrm{H}), 9.81$ (br s, $\left.2 \mathrm{H}, 2 \mathrm{NH}-11\right), 10.15$ (br s, $2 \mathrm{H}, 2 \mathrm{NH}-$ 1), 10.81 (br s, $2 \mathrm{H}, 2 \mathrm{NH}-3$ ) ppm. MS (El, $70 \mathrm{eV}$ ): m/z $820[\mathrm{M}]^{+}$. Anal. calcd for $\mathrm{C}_{48} \mathrm{H}_{32} \mathrm{~N}_{6} \mathrm{O}_{8}: \mathrm{C}, 70.24 ; \mathrm{H}, 3.93 ; \mathrm{N}$, 10.24. Found: C, 70.11; H, 3.78; N, 10.08\%.

\section{Acknowledgements}

Prof. Ismail A. Abdelhamid, Prof. Ahmed H. M. Elwahy and Dr. Amr M. Abdelmoniem acknowledge the Alexander von Humboldt Foundation for scientific fellowships.

\section{References}

1. Fathalla, M.; Lawrence, C. M.; Zhang, N.; Sessler, J. L.; Jayawickramarajah, J. Chem. Soc. Rev. 2009, 38, 1608. https://doi.org/10.1039/b806484a

2. Sivakova, S.; Rowan, S. J. Chem. Soc. Rev. 2005, 34, 9. https://doi.org/10.1039/b304608g

3. Parker, J. B.; Bianchet, M. A.; Krosky, D. J.; Friedman, J. I.; Amzel, L. M.; Stivers, J. T. Nature 2007, 449433. 
https://doi.org/10.1038/nature06131

4. Okamoto, A. Org. Biomol. Chem. 2009, 7, 21.

https://doi.org/10.1039/B813595A

5. McCarthy, O.; Musso-Buendia, A.; Kaiser, M.; Brun, R.; Ruiz-Perez, L. M.; Johansson, N. G.; Pacanowska, D. G.; Gilbert, I. H. Eur. J. Med. Chem. 2009, 44, 678.

https://doi.org/10.1016/j.ejmech.2008.05.018

6. Nair, V.; Chi, G.; Shu, Q.; Julander, J.; Smee, D. F. Bioorg. Med. Chem. Lett. 2009, 19, 1425. https://doi.org/10.1016/j.bmcl.2009.01.031

7. Samanta, A.; Leonidas, D. D.; Dasgupta, S.; Pathak, T.; Zographos, S. E.; Oikonomakos, N. G. J. Med. Chem. 2009, 52, 932.

https://doi.org/10.1021/im800724t

8. Rico-Gómez, R.; López-Romero, J. M.; Hierrezuelo, J.; Brea, J.; Loza, M. I.; Pérez-González, M. Carbohydr. Res. 2008, 343, 855.

https://doi.org/10.1016/j.carres.2008.01.011

9. Tucci, F. C.; Zhu, Y.-F.; Guo, Z.; Gross, T. D.; Connors, P. J.; Gao, Y.; Rowbottom, M. W.; Struthers, R. S.; Reinhart, G. J.; Xie, Q.; Chen, T. K.; Bozigian, H.; Bonneville, A. L. K.; Fisher, A.; Jin, L.; Saunders, J.; Chen, C. J. Med. Chem. 2004, 47, 3483.

https://doi.org/10.1021/im049791w

10. Sutherlin, D. P.; Sampath, D.; Berry, M.; Castanedo, G.; Chang, Z.; Chuckowree, I.; Dotson, J.; Folkes, A.; Friedman, L.; Goldsmith, R.; Heffron, T.; Lee, L.; Lesnick, J.; Lewis, C.; Mathieu, S.; Nonomiya, J.; Zhu, B.-Y. J. Med. Chem. 2010, 53, 1086.

https://doi.org/10.1021/jm901284w

11. Strobel, S.; Dervan, P.; Berg, R.; Buchardt, O. Science 1990, 249, 73.

https://doi.org/10.1126/science.2195655

12. Bansal, R.; Kumar, G.; Gandhi, D.; Young, L. C.; Harvey, A. L. Eur. J. Med. Chem. 2009, 44, 2122. https://doi.org/10.1016/i.ejmech.2008.10.017

13. Drabczyńska, A.; Müller, C. E.; Schiedel, A.; Schumacher, B.; Karolak-Wojciechowska, J.; Fruziński, A.; Zobnina, W.; Yuzlenko, O.; Kieć-Kononowicz, K. Bioorg. Med. Chem. 2007, 15, 6956.

https://doi.org/10.1016/j.bmc.2007.07.051

14. Thureau, P.; Ancian, B.; Viel, S.; Thévand, A. Chem. Commun. 2006, 2006, 200. https://doi.org/10.1039/B513580J

15. Thakur, A. J.; Saikia, P.; Prajapati, D.; Sandhu, J. S. Synlett 2001, 1299. https://doi.org/10.1055/s-2001-16036

16. Agarwal, A.; Chauhan, P. M. S. Tetrahedron Lett. 2005, 46, 1345. https://doi.org/10.1016/j.tetlet.2004.12.109

17. Shi, D.-Q.; Niu, L.-H.; Yao, H.; Jiang, H. J. Heterocycl. Chem. 2009, 46, 237. https://doi.org/10.1002/jhet.57

18. Edjlali, L.; Khanamiri, R. H.; Abolhasani, J. Monatsh. Chem. 2015, 146, 1339. https://doi.org/10.1007/s00706-014-1368-5

19. Shi, D.-Q.; Ni, S.-N.; Yang, F.; Shi, J.-W.; Dou, G.-L.; Li, X.-Y.; Wang, X.-S.; Ji, S.-J. J. Heterocycl. Chem. 2008, $45,693$. https://doi.org/10.1002/ihet.5570450310

20. Tanifum, E. A.; Kots, A. Y.; Choi, B.-K.; Murad, F.; Gilbertson, S. R. Bioorg. Med. Chem. Lett. 2009, $19,3067$. https://doi.org/10.1016/j.bmcl.2009.04.024 
21. Tu, S.; Fang, F.; Li, T.; Zhu, S.; Zhang, X. J. Heterocycl. Chem. 2005, $42,707$. https://doi.org/10.1002/jhet.5570420436

22. Ghorab, M.; Ragab, F.; Noaman, E.; Heiba, H.; El-Hossary, E. Arzneimittelforschung 2011, $57,795$. https://doi.org/10.1055/s-0031-1296682

23. Ghorab, M. M.; Ragab, F. a; Heiba, H. I.; Arafa, R. K.; El-Hossary, E. M. Eur. J. Med. Chem. 2010, $45,3677$. https://doi.org/10.1016/j.ejmech.2010.05.014

24. Candeias, N. R.; Montalbano, F.; Cal, P. M. S. D.; Gois, P. M. P. Chem. Rev. 2010, 110, 6169. https://doi.org/10.1021/cr100108k

25. Isambert, N.; Duque, M. del M. S.; Plaquevent, J.-C.; Génisson, Y.; Rodriguez, J.; Constantieux, T. Chem. Soc. Rev. 2011, 40, 1347.

https://doi.org/10.1039/COCS00013B

26. Shiri, M. Chem. Rev. 2012, 112, 3508. https://doi.org/10.1021/cr2003954

27. Dömling, A.; Wang, W.; Wang, K. Chem. Rev. 2012, 112, 3083. https://doi.org/10.1021/cr100233r

28. Brauch, S.; van Berkel, S. S.; Westermann, B. Chem. Soc. Rev. 2013, 42, 4948. https://doi.org/10.1039/c3cs35505e

29. Abdella, A. M.; Elwahy, A. H. M.; Abdelhamid, I. A. Curr. Org. Synth. 2016, 13 (4), 601. https://doi.org/10.2174/1570179413999151211115100

30. Diab, H. M.; Abdelhamid, I. A.; Elwahy, A. H. M. Synlett 2018, 1627. https://doi.org/10.1055/s-0037-1609967

31. Sanad, S. M. H.; Kassab, R. M.; Abdelhamid, I. A.; Elwahy, A. H. M. Heterocycles 2016, 92, 910. https://doi.org/10.3987/COM-16-13441

32. Abdella, A. M.; Mohamed, M. F.; Mohamed, A. F.; Elwahy, A. H. M.; Abdelhamid, I. A. J. Heterocycl. Chem. 2018, 55 (2), 498. https://doi.org/10.1002/ihet.3072

33. Abdelmoniem, A. M.; Ghozlan, S. A. S.; Abdelmoniem, D. M.; Elwahy, A. H. M.; Abdelhamid, I. A. J. Heterocycl. Chem. 2017, 54, 2844.

https://doi.org/10.1002/jhet.2890

34. Salama, S. K.; Mohamed, M. F.; Darweesh, A. F.; Elwahy, A. H. M.; Abdelhamid, I. A. Bioorg. Chem. 2017, $71,19$. https://doi.org/10.1016/i.bioorg.2017.01.009

35. Hebishy, A. M. S.; Abdelhamid, I. A.; Elwahy, A. H. M. Arkivoc 2018, (5) 97. https://doi.org/10.24820/ark.5550190.p010.367

36. Mohamed, M. F.; Darweesh, A. F.; Elwahy, A. H. M.; Abdelhamid, I. A. RSC Adv. 2016, 6, 40900. https://doi.org/10.1039/C6RA04974E

37. Abdelhamid, I. A.; Darweesh, A. F.; Elwahy, A. H. M. Tetrahedron Lett. 2015, 56, 7085 https://doi.org/10.1016/i.tetlet.2015.11.015 